\title{
Exact Histogram Specification for Digital Images Using a Variational Approach
}

\author{
Mila Nikolova, You-Wei Wen, Raymond Chan
}

\begin{abstract}
We consider the problem of exact histogram specification for digital (quantized) images. The goal is to transform the input digital image into an output (also digital) image that follows a prescribed histogram. Classical histogram modification methods are designed for real-valued images where all pixels have different values, so exact histogram specification is straightforward. Digital images typically have numerous pixels which share the same value. If one imposes the prescribed histogram to a digital image, usually there are numerous ways of assigning the prescribed values to the quantized values of the image. Therefore, exact histogram specification for digital images is an ill-posed problem. In order to guarantee that any prescribed histogram will be satisfied exactly, all pixels of the input digital image must be rearranged in a strictly ordered way. Further, the obtained strict ordering must faithfully account for the specific features of the input digital image. Such a task can be realized if we are able to extract additional representative information (called auxiliary attributes) from the input digital image. This is a real challenge in exact histogram specification for digital images. We propose a new method that efficiently provides a strict and faithful ordering for all pixel values. It is based on a well designed variational approach. Noticing that the input digital image contains quantization noise, we minimize a specialized objective function whose solution is a real-valued image with slightly reduced quantization noise, which remains very close to the input digital image. We show that all the pixels of this real-valued image can be ordered in a strict way with a very high probability. Then transforming the latter image into another digital image satisfying a specified histogram is an easy task. Numerical results show that our method outperforms by far the existing competing methods.
\end{abstract}

Key words: Exact histogram specification, strict-ordering, variational methods, restoration from quantization noise, smooth nonlinear optimization, convex minimization, perturbation analysis.

\section{INTRODUCTION}

The histogram of an image counts the number of pixels at each different intensity value. Image histogram processing alters the value of each individual pixel in order to modify the appearance of the whole image. It is one of the most important image processing tools with various applications, such as contrast enhancement [12], [34], [38], [58], [59], [62], segmentation [13], [44], watermarking [16], texture synthesis [53], texture processing [22], [41] and pattern recognition [39], among many others.

One of the basic histogram processing tasks is histogram equalization [27], [50], [55]. It aims to find a transformation so that the output image has a uniform histogram. More generally, given an input image, histogram specification or histogram matching is the problem to transform it into a similar image that has a

M. Nikolova is with Centre de Mathématiques et de Leurs Applications (CMLA), ENS Cachan, CNRS, 61 av. du Président Wilson, 94235 Cachan Cedex, France. E-mail: nikolova@cmla.ens-cachan.fr.

Y.-W. Wen is with Faculty of Science, Kunming University of Science and Technology, Yunnan, China. The research was supported in part by NFSC Grant. E-mail: wenyouwei@gmail.com.

R. Chan is with Department of Mathematics, The Chinese University of Hong Kong, Shatin, Hong Kong. The research was supported in part by HKRGC Grant CUHK400412 and DAG Grant 2060408. E-mail: rchan@math. cuhk. edu . hk. 


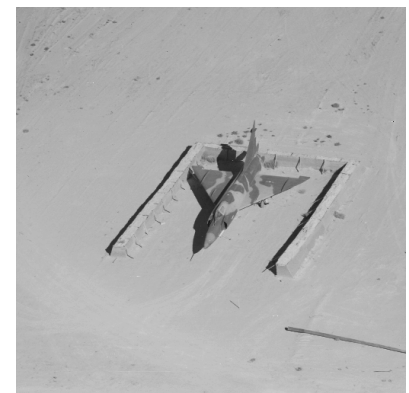

(a) Digital image

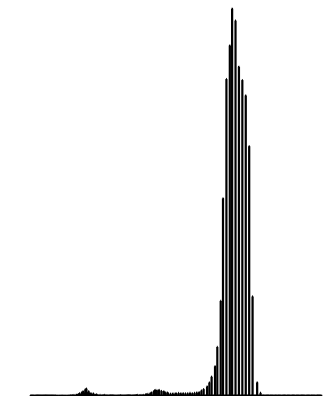

(b) Histogram of (a)

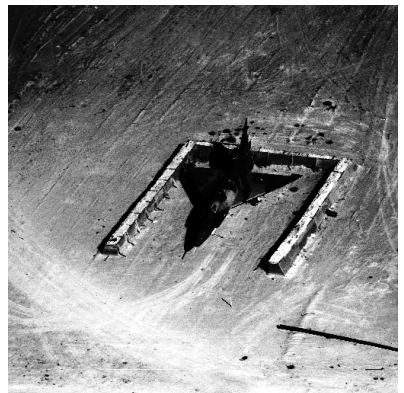

(c) Equalized via histeq.m

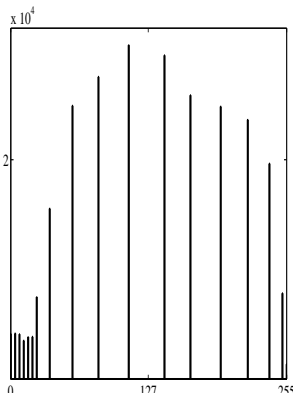

Histogram of (c)

Fig. 1: The original image (a) has gray values in $\{0, \cdots, 255\}$ and $11.62 \%$ of its pixels equal to 177 (the maximum of the histogram in (b)). The built-in Matlab function for histogram equalization (histeq.m) yields the image shown in (c) whose histogram, plotted in (d), is far from being uniform.

pre-specified histogram shape. A desired histogram can be specified according to various needs. Numerous methods have been proposed to improve the histogram of an input image. The simplest one is histogram linear stretching [43]. Histogram clipping [52] limits the maximum number of pixels for each intensity level to a given constant and the clipped pixels are then uniformly distributed among the other intensity levels where the numbers of pixels are less than the clip limit. Several other methods were proposed to preserve the mean brightness of the input image [12], [34], [62]. The prescription of a histogram by combining two histograms was considered in [18], [19]. Sapiro and Caselles [56] proposed histogram modification via image evolution equations. Arici et al. exhibited a general framework for histogram modification [1]. Based on perceptual consideration, Sen and Sankar [57] established a rule enabling an optimal histogram to be prescribed based on the histogram of the input image.

The principle behind histogram specification is straightforward for real-valued (analog) images [55]: the histogram of the input image and the prescribed histogram should be equalized to uniform distribution first, say by $T_{i}$ and $T_{t}$ respectively. Then the output image can be obtained from the composite transformation $T_{t}^{-1} \circ T_{i}$. Since the images are real-valued, $T_{i}$ and $T_{t}$ are one-to-one functions, and hence $T_{t}^{-1} \circ T_{i}$ is welldefined. The principle fails, however, for quantized (digital) images, which is the case of all digital imaging systems. The reason is that for quantized images, the intensity levels of all pixels take a limited number of discrete values (e.g. 256) which is much smaller than the number of the pixels in the image (e.g. $1024^{2}$ ). Therefore exact histogram equalization is hopeless, see Fig. 1. It is only when one can order the pixels of a quantized image in a strict way that the histogram specification problem can be solved exactly for any prescribed histogram.

Several approaches to obtain strict ordering for digital (quantized) images were considered in the literature. This problem is the main goal of our paper. Random ordering of the pixels sharing the same intensity level is the simplest way to do the job [51]. One way of avoiding randomness is to separate pixels having the same intensity using the local mean of the four nearest neighbors as was proposed in [29]. The most successful contemporary methods are the Local Mean (LM) approach proposed by Coltuc et al. [17] and the waveletbased approach (WA) elaborated by Wan and Shi in [61]. More details on these methods are given in Section II. Nonetheless, a major drawback of both the LM and the WA methods is that in practice, they can order strictly only a limited portion of the pixels, and this set of pixels is smaller for images whose histogram is not that uniform to begin with.

In this paper, we propose a specialized variational method that enables us to order in a strict way the pixel 
values of a digital image by slightly reducing the quantization noise that they contain. A sketch of our approach was given in a conference paper [11]. Here we provide a theoretical analysis of our variational method and show that the pixels of the images that it yields can be ordered in a strict way with a high probability. What is more, we ensure that these images are really very close to the input digital image since the $\ell_{\infty}$-norm of the residual is fixed to a suitably small number. We present a lot of experimental results demonstrating that our method outperforms by far its main competitors-the local mean [17] and the wavelet-based [61] methods - both in terms of strict ordering of the pixels and of the quality of the output images.

The outline of the paper is as follows. In Section II, we give comments on sorting algorithm for exact histogram specification. Section III is devoted to the minimizers of the proposed variational functional. In Section IV, we summarize the algorithm for exact histogram specification. In Section V numerical tests are provided where our algorithm is compared with the local mean and wavelet-based approach methods. Concluding remarks are given in Section VI.

\section{Sorting Algorithms}

Let $\mathbf{u}$ be an $M$-by- $N$ image obtained by digitizing an analog image $\mathbf{u}_{o}$ (on a discrete grid) with range in some interval $[0, a)$. We assume the possible quantized values that $\mathbf{u}$ can take are from

$$
\mathcal{P} \stackrel{\text { def }}{=}\left\{p_{1}, \cdots, p_{L}\right\}
$$

and that $p_{k}$ are in increasing order. For 8-bit images, $\mathcal{P}=\{0, \cdots, 255\}$. In the following we will express $\mathbf{u}$ as an $n$-vector by concatenating the columns in $\mathbf{u}$, hence $n=M \times N$. Denote $\mathbb{I}_{q} \stackrel{\text { def }}{=}\{1, \ldots, q\}$ for any positive integer $q$ and $\Omega_{k} \stackrel{\text { def }}{=}\left\{i \in \mathbb{I}_{n} \mid \mathbf{u}[i]=p_{k}\right\}, k=1,2, \cdots, L$. The histogram of $\mathbf{u}$ is the $L$-tuple $\mathbf{h}_{\mathbf{u}}=\left(\left|\Omega_{1}\right|,\left|\Omega_{2}\right|, \ldots,\left|\Omega_{L}\right|\right)$, where $|\cdot|$ stands for cardinality. The image $\mathbf{u}$ with histogram $\mathbf{h}_{\mathbf{u}}$ is a result of quantization of the original real-valued image $\mathbf{u}_{o}$. This amounts to set to the same intensity level $p_{k}$ all the values of $\mathbf{u}_{o}$ on the interval $\left[t_{k-1}, t_{k}\right), k=1, \ldots, L$. Then $\left|\Omega_{k}\right|=n \int_{t_{k-1}}^{t_{k}} p\left(\mathbf{u}_{o}\right) d t$, where $p\left(\mathbf{u}_{o}\right)$ is the probability density function of the intensity levels of the pixels of $\mathbf{u}_{o}$, and $t_{0}=0$ and $t_{L}=a$.

Let the pre-specified histogram be $\mathbf{h}=\left(h_{1}, h_{2}, \ldots, h_{L}\right)$. The classical way of defining exact histogram specification is designed for real-value images on a discrete grid like $\mathbf{u}_{o}$. Such images are almost nowhere constant [28] and exact histogram specification is straightforward. However, in practice, we only have the quantized image $\mathbf{u}$, obtained from an original real-valued image $\mathbf{u}_{o}$. The digital $\mathbf{u}$ can seldom be totallyordered-its pixels have only $L$ possible magnitudes while the number of its pixels $n$ is generally much larger, i.e. $n \gg L$. A typical example is shown in Fig. 1. It is therefore very likely that some $\Omega_{k}, k=1, \ldots, L$, will meet $\left|\Omega_{k}\right|>h_{k}$. In order to satisfy the prescribed histogram, some pixels in $\Omega_{k}$ will have values mapped to other intensity levels. The number of ways of selecting the pixels to assign to other intensity levels is very large. Consequently, this problem is ill-posed.

The key to a stable solution is to create a total ordering of the pixels in the same $\Omega_{k}$ by learning some auxiliary information from the digital image $\mathbf{u}$. Suppose that for any pixel $i \in \mathbb{I}_{n},(K-1)$ auxiliary information $\kappa_{1}[i], \ldots, \kappa_{K-1}[i]$ are created. Then an ascending order " $\prec$ " for all pixels in $\mathbb{I}_{n}$ may be obtained using the $K$-tuples

$$
\left(\mathbf{u}[i], \kappa_{1}[i], \ldots, \kappa_{K-1}[i]\right), \quad \forall i \in \mathbb{I}_{n} .
$$

To facilitate the discussions, we set $\kappa_{0}[i] \stackrel{\text { def }}{=} \mathbf{u}[i]$. For any two pixels $i$ and $j$ in $\mathbb{I}_{n}$, we say that $i \prec j$ if for some $-1 \leq \ell<(K-1)$ we have

$$
\kappa_{s}[i]=\kappa_{s}[j] \text { for all } 0 \leq s \leq \ell \text { and } \kappa_{\ell+1}[i]<\kappa_{\ell+1}[j] .
$$


If the auxiliary information $K$ is well chosen, one should be able to sort all pixels $i \in \mathbb{I}_{n}$ in a real-world digital image. That is, we can order all the pixels $i$ in $\mathbb{I}_{n}$ in such a way that $i_{1} \prec i_{2} \prec \ldots \prec i_{n}$. Once such a strict-ordering is obtained, matching the input histogram to the prescribed one is straightforward. This can be done by dividing the ordered list $\left\{i_{\ell}\right\}_{\ell=1}^{n}$ from left to right into $L$ groups. Thus the first $h_{1}$ pixels $i_{1}, i_{2}, \ldots, i_{h_{1}}$ belong to the first group, and are assigned with the intensity of $p_{1}$. The next $h_{2}$ pixels $i_{h_{1}+1}, \ldots, i_{h_{1}+h_{2}}$ belong to the second group and are assigned the intensity of $p_{2}$, and so on until all pixels are assigned to their new intensities.

The Local Mean (LM) and the Wavelet Approach (WA), mentioned in the Introduction, extract such auxiliary information from the input quantized image. The LM approach of Coltuc et al. [17] uses the average intensities of neighboring pixels: given two pixels with the same intensity value, the means over the neighborhoods centered at each pixel are compared to order these pixels. If the mean values are still the same, then larger neighborhoods are chosen to compute the average intensity. This procedure is repeated until all pixels are ordered. The authors claim that $K=6$ is "appropriate for any application" [17]. Wan and Shi argue that the LM approach fails to keep sharp the edges in the output image [61]. They proposed to order the pixels according to the absolute values of its wavelet coefficients. This approach tends to amplify any noise since noise in a smooth region may be mistaken as an edge and hence is sharpened. Post-processing approach or iterative methods can be applied to suppress the amplified noises [3].

\section{A SPECIALIZED VARIATIONAL APPROACH}

We use a different paradigm to obtain auxiliary information. The available image $\mathbf{u}$ contains quantization noise. Our strategy is to built a real-valued image where this noise is slightly reduced. Even though the original real-valued $\mathbf{u}_{o}$ is unknown, some general priors such as the presence of edges and fine structures in $\mathbf{u}_{o}$ can be employed to produce such a restored version of $\mathbf{u}$. A subtle task like this can be handled by means of a well conceived variational method.

Given $\mathbf{u}$, its real-valued version $\widehat{\mathbf{f}}$ is defined as the minimizer $\arg \min _{\mathbf{f} \in \mathbb{R}^{n}} \mathcal{J}(\mathbf{f}, \mathbf{u})$ of a smooth, convex regularized cost function $\mathcal{J}(\cdot, \mathbf{u}): \mathbb{R}^{n} \rightarrow \mathbb{R}$,

$$
\mathcal{J}(\mathbf{f}, \mathbf{u})=\Psi(\mathbf{f}, \mathbf{u})+\beta \Phi(\mathbf{f}),
$$

where both the data fitting term $\Psi$ and the regularization term $\Phi$ are asymptotically nearly affine. Here $\beta>0$ is a weighting parameter. These terms read

$$
\begin{aligned}
& \Psi(\mathbf{f}, \mathbf{u})=\sum_{i \in \mathbb{I}_{n}} \psi(\mathbf{f}[i]-\mathbf{u}[i]), \\
& \Phi(\mathbf{f}) \quad=\sum_{i \in \mathbb{I}_{n}} \sum_{j \in \mathcal{N}_{i}} \phi(\mathbf{f}[i]-\mathbf{f}[j]),
\end{aligned}
$$

where the functions $\psi(\cdot) \stackrel{\text { def }}{=} \psi\left(\cdot, \alpha_{1}\right): \mathbb{R} \rightarrow \mathbb{R}$ and $\phi(\cdot) \stackrel{\text { def }}{=} \phi\left(\cdot, \alpha_{1}\right): \mathbb{R} \rightarrow \mathbb{R}$ depend on two parameters $\alpha_{1}>0$ and $\alpha_{2}>0$, respectively, and $\mathcal{N}_{i}$ is the set of the four or the eight adjacent neighbors of pixel $i$ in the image, for every $i \in \mathbb{I}_{n}$ (see Fig. 2). The parameters $\alpha_{1}$ and $\alpha_{2}$ shall be omitted when they are not explicitly involved in our derivations.

The functions $\psi$ and $\phi$ in (3) belong to the family of functions $\theta(\cdot, \alpha): \mathbb{R} \rightarrow \mathbb{R}, \alpha>0$, satisfying the conditions $\mathrm{H} 1$ and $\mathrm{H} 2$ described below. 


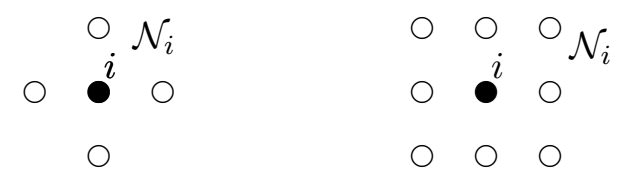

Fig. 2: $\mathcal{N}_{i}$ is the set of the 4 (left) / 8 (right) adjacent pixels of $i$.

H1. The function $\theta(\cdot, \alpha)$ is $\mathcal{C}^{s}$-continuous for $s \geq 2$, even-i.e. $\theta(-t, \alpha)=\theta(t, \alpha)$-and for any $\alpha>0$ its second derivative with respect to $t$ satisfies

$$
t \in \mathbb{R} \quad \Rightarrow \quad \theta^{\prime \prime}(t, \alpha)>0
$$

Note that by $\mathrm{H} 1, t \rightarrow \theta^{\prime}(t, \alpha)$ is strictly increasing in $t$. Further, $\theta(\cdot, \alpha)$ is nearly affine beyond a small neighborhood of the origin. More precisely,

H2. The first derivative of $\theta(\cdot, \alpha)$ with respect to $t$ is upper bounded for $\alpha>0$ fixed and is strictly decreasing in $\alpha>0$ for any given $t>0$ :

$$
\begin{array}{ll}
\alpha>0 & \Rightarrow \quad \lim _{t \rightarrow \infty} \theta^{\prime}(t, \alpha)=1, \\
t \in \mathbb{R} & \Rightarrow \quad \lim _{\alpha \rightarrow 0} \theta^{\prime}(t, \alpha)=1 \quad \text { and } \quad \lim _{\alpha \rightarrow \infty} \theta^{\prime}(t, \alpha)=0 .
\end{array}
$$

Good choices for $\theta$ meeting $\mathrm{H} 1$ and $\mathrm{H} 2$ are given in Table I. Customarily, such functions are involved only in the regularization term, combined with a quadratic data-fidelity term, see e.g. [8], [2]. In order to achieve our objectives, they are pertinent to define the data fitting term as well.

\begin{tabular}{|l||c|c|c|}
\hline & $\theta$ & $\theta^{\prime}$ & $\theta^{\prime \prime}$ \\
\hline f1 & $\sqrt{t^{2}+\alpha}$ & $\frac{t}{\sqrt{t^{2}+\alpha}}$ & $\frac{\alpha}{\left(\sqrt{t^{2}+\alpha}\right)^{3}}$ \\
\hline f2 & $\alpha \log \left(\cosh \left(\frac{t}{\alpha}\right)\right)$ & $\tanh \left(\frac{t}{\alpha}\right)$ & $\frac{1}{\alpha}\left(1-\left(\tanh \left(\frac{t}{\alpha}\right)\right)^{2}\right)$ \\
\hline f3 & $|t|-\alpha \log \left(1+\frac{|t|}{\alpha}\right)$ & $\frac{t}{\alpha+|t|}$ & $\frac{\alpha}{(\alpha+|t|)^{2}}$ \\
\hline
\end{tabular}

TABLE I: Relevant choices for $\theta(\cdot, \alpha)$ obeying $\mathrm{H} 1$ and $\mathrm{H} 2$. The size of the neighborhood of zero where these functions are not "nearly affine" is controlled by the parameter $\alpha>0$.

The graphs of $\theta$ and $\theta^{\prime}$ for two functions in Table I for two different values of $\alpha$ are shown in Fig. 3 .
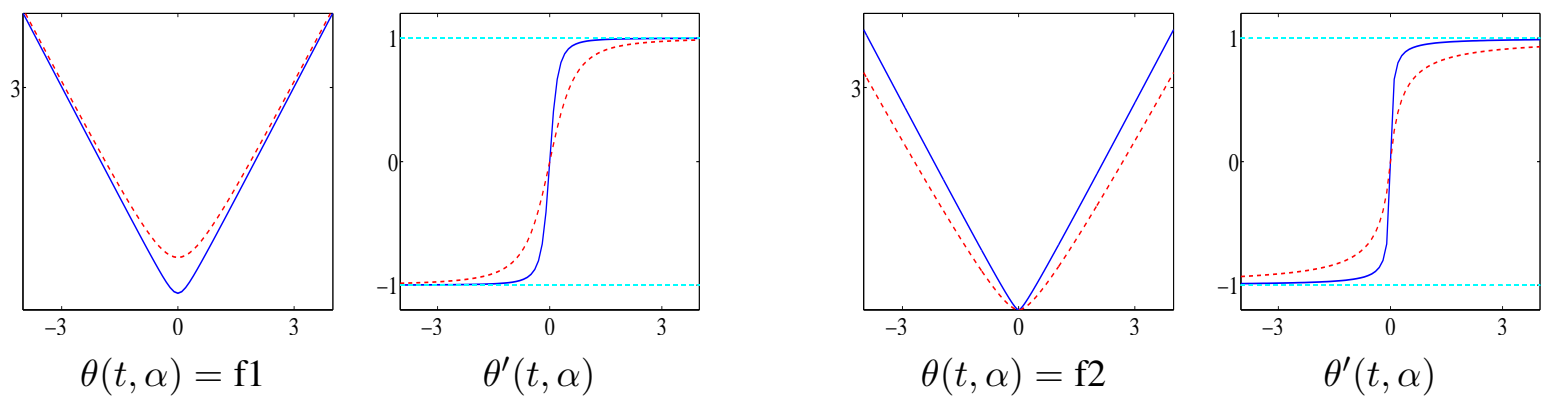

Fig. 3: The first two functions in Table I where the plots for $\alpha=0.05$ are in blue solid curves and the plots for $\alpha=0.5$ are in red dashed curves. 
Remark (a): Motivation to choose a $\mathcal{J}$ satisfying $H 1$ and H2. Before going into the details, we explain the intuition behind the demands $\mathrm{H} 1$ and $\mathrm{H} 2$ addressed to $\mathcal{J}$ in (2). Our main concern is to obtain a restoration $\widehat{\mathbf{f}}$ of $\mathbf{u}$ whose pixels are all different from each other while being close to $\mathbf{u}$ but "better" than $\mathbf{u}$. Since $\phi$ satisfies $\mathrm{H} 1$, we will get minimizers $\widehat{\mathbf{f}}$ that are generically nowhere constant [47]. Rather, they present large variations, edges and fine structures. The trend of the large variations is available in the input image $\mathbf{u}$. Requirement $\mathrm{H} 2$ on $\phi$ enables the recovery of edges and details and in this way some removal of the quantization noise. For instance, $\alpha_{2}>0$ should be just small enough. Selecting a small $\beta>0$ (compared to the range of $\mathbf{u}$ ) enhances fitting to data $\mathbf{u}$. If the neighborhood near the origin where $\psi$ is not quasi-affine goes to zero (e.g. $\alpha_{1}$ is almost zero), $\psi$ tends to the absolute value function. The latter is known to generate minimizers $\widehat{\mathbf{f}}$ containing a certain number of entries equal to the relevant entries of $\mathbf{u}$ [46]. These minimizers may still contain numerous equally valued pixels, hence such a scenario must be avoided. If $\psi$ obeys $\mathrm{H} 1$ and $\mathrm{H} 2$ (in words, we should take $\alpha_{1}>0$ small enough), the components of $\widehat{\mathbf{f}}$ will certainly be close but different from the relevant entries of $\mathbf{u}$. So the level sets of the digital image $\mathbf{u}$ are not destroyed, but only refined. This is the reason why we can say that details are preserved in $\widehat{\mathbf{f}}$. Undoubtedly, pixels in $\widehat{\mathbf{f}}$ must change from those in $\mathbf{u}$ no more than a given value (for example $|0.1|$ ). The soundness of these arguments is illustrated in Fig. 4. We can thus claim that the objective $\mathcal{J}$ defined using (2) and (3), where $\psi$ and $\phi$ satisfy $\mathrm{H} 1$ and $\mathrm{H} 2$ is detail preserving. Rigorous facts on this point are presented in subsection III-C.

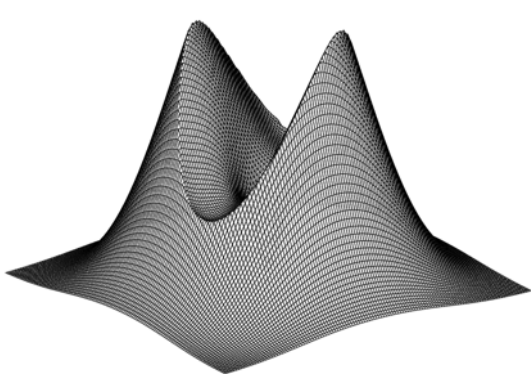

(a) Real-valued $\mathbf{u}_{o}$

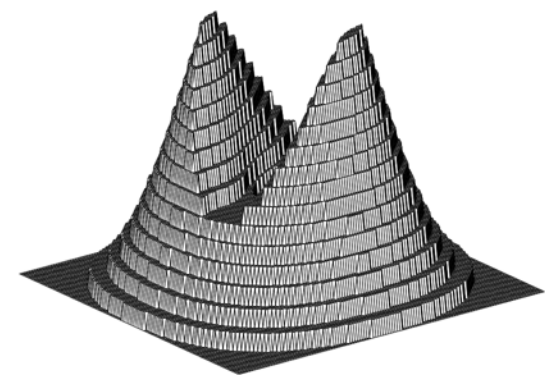

(b) $\mathbf{u}$ quantized on $\{0, \cdots, 15\}$

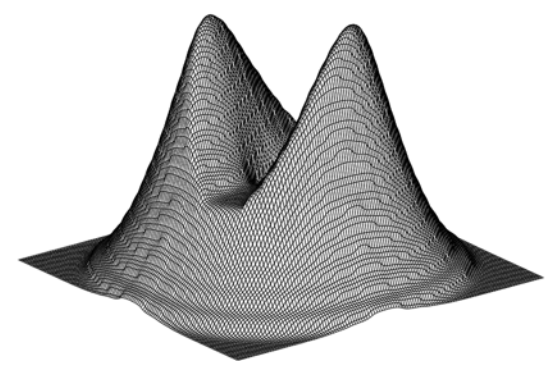

(c) Restored $\widehat{\mathbf{f}}$

Fig. 4: The restored $\widehat{\mathbf{f}}$ in (c) is obtained by minimizing $\mathcal{J}(\cdot, \mathbf{u})$ where $\psi(t)=\sqrt{\alpha_{1}+t^{2}}$ and $\phi(t)=\sqrt{\alpha_{2}+t^{2}}$.

Below we exhibit other salient properties of the minimizer $\widehat{\mathbf{f}}$ of $\mathcal{J}$ under H1 and $\mathrm{H} 2$ that enable a reasonable strict ordering of the pixels of real-world digital images. It is worth noticing that they are independent of the particular shapes of $\psi$ and $\phi$, provided that they meet $\mathrm{H} 1$ and $\mathrm{H} 2$. The latter remark presents a challenging topic for future research.

\section{A. Preliminary results}

The differences $\left\{\mathbf{f}[i]-\mathbf{f}[j] \mid j \in \mathcal{N}_{i}\right\}$ for all $i \in \mathbb{I}_{n}$ in (3) can be rewritten using finite difference operators $\mathbf{g}_{i} \in \mathbb{R}^{n}, 1 \leq i \leq r$, where $r$ is the total number of these operators. Then $\Phi$ reads

$$
\Phi(\mathbf{f})=\sum_{i \in \mathbb{I}_{r}} \phi\left(\mathbf{g}_{i}^{T} \mathbf{f}\right)
$$

Let us denote

$$
G=\left[\begin{array}{c}
\mathbf{g}_{1}^{T} \\
\vdots \\
\mathbf{g}_{r}^{T}
\end{array}\right] \in \mathbb{R}^{r \times n}
$$


According to (3) and the adopted boundary conditions (Neumann), we have $\operatorname{ker} G=\{c \mathbb{1} \mid c \in \mathbb{R}\}$, where 11 is a vector composed of ones.

We shall study how the minimizer $\widehat{\mathbf{f}}$ of $\mathcal{J}$ behaves as a function of data $\mathbf{u}$. This goal motivates the definition below which is originally introduced in [45]. For clarity, it is restated in a way adapted to this work.

Definition 1. A function $\mathcal{F}: \mathcal{O} \rightarrow \mathbb{R}^{n}$, where $\mathcal{O}$ is an open domain in $\mathbb{R}^{n}$, is said to be a minimizer function relevant to the family of functions $\mathcal{J}(\cdot, \mathcal{O})$ if for every $\mathbf{u} \in \mathcal{O}$, the point $\widehat{\mathbf{f}}=\mathcal{F}(\mathbf{u})$ is a strict local minimizer of $\mathcal{J}(\cdot, \mathbf{u})$.

The next lemma is a straightforward extension of the Implicit Functions Theorem [4]. Its proof can be found e.g. in [23, Theorem 6, p. 34] or in [33, Lemma 6.1.1, p. 268]. In what follows, $D_{i}^{j}$ stands for the $j$ th order differential of a function with respect to the $i$ th variable ${ }^{1}$.

Lamma 1. Suppose that $\mathcal{J}: \mathbb{R}^{n} \times \mathbb{R}^{n} \rightarrow \mathbb{R}$ is any function which is $\mathcal{C}^{s}$, with $s \geq 2$. Fix $\mathbf{u} \in \mathbb{R}^{n}$. Let $\widehat{\mathbf{f}} \in \mathbb{R}^{n}$ be such that $D_{1} \mathcal{J}(\widehat{\mathbf{f}}, \mathbf{u})=0$ and $D_{1}^{2} \mathcal{J}(\widehat{\mathbf{f}}, \mathbf{u})$ is positive definite. Then there exists an open neighborhood of $\mathbf{u}$, say $\mathcal{O}$, and a unique $\mathcal{C}^{s-1}$ minimizer function $\mathcal{F}: \mathcal{O} \rightarrow \mathbb{R}^{n}$ such that $\mathcal{F}(\mathbf{u})=\widehat{\mathbf{f}}$.

We will see that in our case, the minimizer function is uniquely defined on $\mathbb{R}^{n}$.

Proposition 1. Let $\mathcal{J}: \mathbb{R}^{n} \times \mathbb{R}^{n} \rightarrow \mathbb{R}$ in (2) satisfy H1. Then for any $\beta>0, \mathcal{J}$ has a unique minimizer function $\mathcal{F}: \mathbb{R}^{n} \rightarrow \mathbb{R}^{n}$ which is $\mathcal{C}^{s-1}$ continuous.

The proof of the proposition is given in Appendix VII-A. The components of the minimizer function $\mathcal{F}$ read $\mathcal{F}_{i}, i \in \mathbb{I}_{n}$. A diagonal matrix $A$ with diagonal entries $a[i], i \in \mathbb{I}_{n}$, is denoted by $\left.A=\operatorname{diag}(\{a[i])\}_{i=1}^{n}\right)$.

Lamma 2. Let $\beta>0$ be arbitrary and $\mathcal{J}: \mathbb{R}^{n} \times \mathbb{R}^{n} \rightarrow \mathbb{R}$ in (2) satisfy H1. Then its Hessian matrix $H(\mathbf{u}) \stackrel{\text { def }}{=} D_{1}^{2} \mathcal{J}(\mathcal{F}(\mathbf{u}), \mathbf{u})$, given by

$$
H(\mathbf{u})=\operatorname{diag}\left(\left\{\psi^{\prime \prime}\left(\mathcal{F}_{i}(\mathbf{u})-\mathbf{u}[i]\right)\right\}_{i=1}^{n}\right)+\beta G^{T} \operatorname{diag}\left(\left\{\phi^{\prime \prime}\left(\mathbf{g}_{i}^{T} \mathcal{F}(\mathbf{u})\right)\right\}_{i=1}^{r}\right) G
$$

is invertible. Consequently, the differential $D \mathcal{F}: \mathbb{R}^{n} \rightarrow \mathbb{R}^{n}$ of the minimizer function $\mathcal{F}$ of $\mathcal{J}$ reads

$$
D \mathcal{F}(\mathbf{u})=\left[\begin{array}{c}
D \mathcal{F}_{1}(\mathbf{u}) \\
\cdots \\
D \mathcal{F}_{p}(\mathbf{u})
\end{array}\right]=(H(\mathbf{u}))^{-1} \operatorname{diag}\left(\left\{\psi^{\prime \prime}\left(\mathcal{F}_{i}(\mathbf{u})-\mathbf{u}[i]\right)\right\}_{i=1}^{n}\right)
$$

and satisfies

$$
\operatorname{rank}(D \mathcal{F}(\mathbf{u}))=n, \quad \forall \mathbf{u} \in \mathbb{R}^{n}
$$

This simple lemma, proven in Appendix VII-B, underlies the main theoretical results in this work.

\section{B. Crucial features of the minimizers of $\mathcal{J}(\cdot, \mathbf{u})$ and discussion}

First, we show that the minimizer function $\mathcal{F}$ is non-expansive.

Proposition 2. Let for an arbitrary $\beta>0$, the cost function $\mathcal{J}: \mathbb{R}^{n} \times \mathbb{R}^{n} \rightarrow \mathbb{R}$ in (2) satisfy H1. Then its minimizer function $\mathcal{F}: \mathbb{R}^{n} \rightarrow \mathbb{R}^{n}$ is nonexpansive:

$$
(\mathbf{u}, \boldsymbol{\zeta}) \in \mathbb{R}^{n} \times \mathbb{R}^{n} \quad \Rightarrow \quad\|\mathcal{F}(\mathbf{u}+\boldsymbol{\zeta})-\mathcal{F}(\mathbf{u})\|_{2} \leq\|\boldsymbol{\zeta}\|_{2}
$$

${ }^{1}$ E.g., $D_{1}^{j} \mathcal{J}$ is the $j$ th differential of $\mathcal{J}$ in (2) with respect $\mathbf{f}$ and $D_{2}^{j} \mathcal{J}$ is with respect to $\mathbf{u}$. 
One can note that $\mathcal{F}$ is Lipschitz with constant 1. The proof is given in Appendix VII-E.

Our main questions are whether or not some entries of a minimizer $\widehat{\mathbf{f}}$ of $\mathcal{J}(\cdot, \mathbf{u})$ can: $(i)$ take equal values and $(i i)$ be equal to some components of the input image $\mathbf{u}$. For $(i)$, we consider the set $\mathcal{G}$ which is composed of all operators that yield the difference between any two pixels in an image:

$$
\mathcal{G} \stackrel{\text { def }}{=} \bigcup_{(i, j) \in \mathbb{I}_{n} \times \mathbb{I}_{n}}\left\{g \in \mathbb{R}^{n} \mid g[i]=-g[j]=1, i \neq j,(i, j) \in \mathbb{I}_{n}, g[k]=0, k \in \mathbb{I}_{n} \backslash(i \cup j)\right\} .
$$

Observe that all difference operators in (4) satisfy $\mathbf{g}_{i} \in \mathcal{G}, \forall i \in \mathbb{I}_{r}$.

Our main result is stated below. Its proof is presented in Appendix VII-C. As usual, the Lebesgue measure on $\mathbb{R}^{n}$ is denoted by $\mathbb{L}^{n}(\cdot)$.

Theorem 1. Let $\mathcal{J}: \mathbb{R}^{n} \times \mathbb{R}^{n} \rightarrow \mathbb{R}$ in (2) satisfy H1. For its minimizer function $\mathcal{F}: \mathbb{R}^{n} \rightarrow \mathbb{R}^{n}$, define the sets $K_{\mathcal{G}}$ and $K_{\mathcal{I}}$ as follows:

$$
\begin{gathered}
K_{\mathcal{G}}=\bigcup_{g \in \mathcal{G}}\left\{\mathbf{u} \in \mathbb{R}^{n} \mid g^{T} \mathcal{F}(\mathbf{u})=0\right\}, \\
K_{\mathcal{I}}=\bigcup_{i \in \mathbb{I}_{n}} \bigcup_{j \in \mathbb{I}_{n}}\left\{\mathbf{u} \in \mathbb{R}^{n} \quad \mid \mathcal{F}_{i}(\mathbf{u})=\mathbf{u}[j]\right\} .
\end{gathered}
$$

Then $K_{\mathcal{G}}$ and $K_{\mathcal{I}}$ are closed in $\mathbb{R}^{n}$ and obey

$$
\mathbb{L}^{n}\left(K_{\mathcal{G}}\right)=0 \quad \text { and } \quad \mathbb{L}^{n}\left(K_{\mathcal{I}}\right)=0 .
$$

The result holds true for any $\beta>0$.

The sets $K_{\mathcal{G}}$ in (9) contains all possible $\mathbf{u} \in \mathbb{R}^{n}$ such that a minimizer $\widehat{\mathbf{f}}=\mathcal{F}(\mathbf{u})$ of $\mathcal{J}(\cdot, \mathbf{u})$ might have two equal entries, $\widehat{\mathbf{f}}[i]=\widehat{\mathbf{f}}[j]$ for some $i \neq j$ where $(i, j) \in \mathbb{I}_{n} \times \mathbb{I}_{n}$. The set $K_{\mathcal{I}}$ in (10) contains all possible $\mathbf{u} \in \mathbb{R}^{n}$ such that the minimizer $\widehat{\mathbf{f}}=\mathcal{F}(\mathbf{u})$ might contain some entries equal to data entries, $\mathcal{F}_{i}(\mathbf{u})=\mathbf{u}[j]$ for some $(i, j) \in \mathbb{I}_{n} \times \mathbb{I}_{n}$.

According to Theorem 1,

- The elements of $K_{\mathcal{G}}$ are highly exceptional in $\mathbb{R}^{n}$. Indeed, the subset $\mathbb{R}^{n} \backslash K_{\mathcal{G}}$ contains an open and dense subset of $\mathbb{R}^{n}$. The chance that a truly random $\mathbf{u} \in \mathbb{R}^{n}$-i.e. a $\mathbf{u}$ following a non-singular probability distribution on $\mathbb{R}^{n}$-happens to be in $K_{\mathcal{G}}$ can be ignored in practice. Conversely, $\mathcal{F}_{i}(\mathbf{u}) \neq \mathcal{F}_{j}(\mathbf{u})$, for $i \neq j$, is a generic property ${ }^{2}$ of the minimizer $\mathcal{F}(\mathbf{u})$ of $\mathcal{J}(\cdot, \mathbf{u})$, as given in (2) and satisfying H1.

- The event $\mathcal{F}_{i}(\mathbf{u})=\mathbf{u}[i]$ is highly exceptional, as anticipated in Remark (a) "Motivation". The minimizer functions $\mathcal{F}: \mathbb{R}^{n} \rightarrow \mathbb{R}^{n}$ relevant to $\mathcal{J}$ generically satisfy $\mathcal{F}_{i}(\mathbf{u}) \neq \mathbf{u}[j]$, for all $(i, j) \in \mathbb{I}_{n} \times \mathbb{I}_{n}$.

These are general results that hold for any real data $\mathbf{u} \in \mathbb{R}^{n}$.

Remark (b): Discussion. Let us denote by $\mathcal{S}_{\mathcal{P}}^{n}$ the set of all $M \times N$ images whose $n=M N$ pixels values belong to the quantization set $\mathcal{P}$ in (1):

$$
\mathcal{S}_{\mathcal{P}}^{n}=\left\{u \in \mathbb{R}^{n} \mid u[i] \in \mathcal{P}, \forall i \in \mathbb{I}_{n}\right\} .
$$

Recall that $|\mathcal{P}|=L$, the cardinality of $\mathcal{S}_{\mathcal{P}}^{n}$ is $\left|\mathcal{S}_{\mathcal{P}}^{n}\right|=L^{n}$. Even though $\left|\mathcal{S}_{\mathcal{P}}^{n}\right|$ is a huge number ${ }^{3}$, the set $\mathcal{S}_{\mathcal{P}}^{n}$ is finite, hence

$$
\mathbb{L}^{n}\left(\mathcal{S}_{\mathcal{P}}^{n}\right)=0
$$

\footnotetext{
${ }^{2}$ Here we use the terminology in [20].

${ }^{3}$ For $512 \times 512$, 8-bits images, this value is $255^{512^{2}}$ - the amount of all $512 \times 512$, 8-bits pictures that people can ever take with their digital cameras.
} 
Hence the question: what can we say about the possible intersections $K_{\mathcal{G}} \cap \mathcal{S}_{\mathcal{P}}^{n}$ and $K_{\mathcal{I}} \cap \mathcal{S}_{\mathcal{P}}^{n}$ ?

Assume that $\mathcal{P}$ is composed of $L$ integers. Let $N_{\mathcal{P}}$ be the set of the functions that map $\mathcal{P}^{n}$ onto $\mathcal{P}$. We would not like that the minimizer function $\mathcal{F}$ has some components belonging to $N_{\mathcal{P}}$. Number theory gives limited answers to the question of the kind of functions being able to be in $N_{\mathcal{P}}$. For $n=1$, every function applied to an integer $u \in \mathcal{P}$ and yielding an integer $\widehat{f}=f(u)$ is of the form

$$
f(u)=\sum_{k \in \mathbb{I}_{u}} b_{k}\left(\begin{array}{l}
u \\
k
\end{array}\right) \quad \text { where } \quad\left(\begin{array}{l}
u \\
k
\end{array}\right)=\frac{u(u-1) \cdots(u-k+1)}{k !},
$$

where all $b_{k}$ are integers. The question was initially posed in [49]. This result along with some refinements can be found in [9]. However, $f(u) \in N_{\mathcal{P}}$ requires also that $f(u) \in \mathcal{P}$, which drastically limits ${ }^{4}$ the functions of this form that fall into $N_{\mathcal{P}}$. In the case of several variables, some polynomial functions may belong to $N_{\mathcal{P}}$, see [10]. More generally, Diophantine equations [30] can also be cast in the class of polynomial functions. Under the (severe) restriction $f(u) \in \mathcal{P}$, some of them also live in $N_{\mathcal{P}}$. To the best of our knowledge, no other families of functions were shown to be able to belong to $N_{\mathcal{P}}$.

However, given the expression for $D \mathcal{F}$ in Lemma 2, it is not difficult to see that no component $\mathcal{F}_{i}$ of our minimizer function $\mathcal{F}$ can have a polynomial expression.

Let $\mathbf{u}=c \mathbb{1}$ for some $c \in \mathcal{P}$. Then for any $\beta>0$, the minimizer $\widehat{\mathbf{f}}$ of $\mathcal{J}(\cdot, \mathbf{u})$ reads $\widehat{\mathbf{f}}=\mathbf{u}$. Indeed, since $c \mathbb{l l} \in \operatorname{ker} G$, we have $\Psi(\mathbf{u}, \mathbf{u})=0$ and $\Phi(\mathbf{u})=\beta r \phi(0)$, so $\mathcal{J}$ reaches its lower bound for $\widehat{\mathbf{f}}=\mathbf{u}$. Hence all constant digital images meet

$$
\{c \mathbb{1} \mid c \in \mathcal{P}\} \in K_{\mathcal{G}} \cap \mathcal{S}_{\mathcal{P}}^{n} \quad \text { and } \quad\{c \mathbb{1} \mid c \in \mathcal{P}\} \in K_{\mathcal{I}} \cap \mathcal{S}_{\mathcal{P}}^{n} .
$$

Consequently,

$$
K_{\mathcal{G}} \cap \mathcal{S}_{\mathcal{P}}^{n} \neq \varnothing \quad \text { and } \quad K_{\mathcal{I}} \cap \mathcal{S}_{\mathcal{P}}^{n} \neq \varnothing
$$

One can ask what histogram modification would be needed for a constant image. However, there are other simple images that belong to $\left(K_{\mathcal{G}} \cup K_{\mathcal{I}}\right) \cap \mathcal{S}_{\mathcal{P}}^{n}$. We can reasonably conjecture the following:

- $K_{\mathcal{G}} \cap \mathcal{S}_{\mathcal{P}}^{n}$ is essentially composed of simple (synthetic, in practice) images. For most of them, if some histogram modification was needed, it should be defined in a proper way.

- The ratios

$$
\frac{\left|\left(K_{\mathcal{G}} \cap \mathcal{S}_{\mathcal{P}}^{n}\right)\right|}{\left|\mathcal{S}_{\mathcal{P}}^{n}\right|} \text { and } \frac{\left|\left(K_{\mathcal{I}} \cap \mathcal{S}_{\mathcal{P}}^{n}\right)\right|}{\left|\mathcal{S}_{\mathcal{P}}^{n}\right|}
$$

should be numbers close to zero.

Being impossible to prove such a conjecture, we cannot guarantee that for any digital image $\mathbf{u} \in \mathcal{P}^{n}$, the entries of the minimizer $\widehat{\mathbf{f}}=\mathcal{F}(\mathbf{u})$ can be ordered in a strictly increasing way. However, the numerical results on 8-bit images in Table V (in section V) shows that our method enables strict ordering for all tested images.

\section{Selection of the parameters}

Objectives $\mathcal{J}$ of the form given by (2)-(3) belong to a wider family of objectives satisfying $\mathrm{H} 1$ and $\mathrm{H} 2$ that were studied in [5]. Below we sketch some of the main results of [5] as far as they are important to tune our

${ }^{4}$ These polynomial functions yield also arbitrarily large values that exceed the bounded set $\mathcal{P}$. 
applications. Using $\mathrm{H} 1$, for any $\alpha_{1}$ fixed, $\psi^{\prime}\left(\cdot, \alpha_{1}\right)$ is odd and strictly increasing on $\mathbb{R}$, hence $t \mapsto \psi^{\prime}\left(t, \alpha_{1}^{10}\right)$ is invertible on $(-1,1)$. So the function

$$
b\left(y, \alpha_{1}\right) \stackrel{\text { def }}{=}\left(\psi^{\prime}\right)^{-1}\left(y, \alpha_{1}\right) \in \mathbb{R}, \quad \forall y \in(-1,+1), \quad \forall \alpha_{1}>0,
$$

is well defined. Combining our assumptions with [5, Remark 1] shows that for any $\alpha_{1}$ fixed, $y \mapsto b\left(y, \alpha_{1}\right)$ is continuous, odd and strictly increasing. Under Neumann, Dirichlet or periodic boundary conditions, $\left|\mathcal{N}_{i}\right|$ is constant for any $i \in \mathbb{I}_{n}$. Let us denote $\eta \stackrel{\text { def }}{=}\left|\mathcal{N}_{i}\right|$. If we consider the four nearest neighbors then $\eta=\mathcal{N}_{i}=4$ and for the eight nearest neighbors, $\eta=8$. Given $\mathbf{u}$, let $\widehat{\mathbf{f}}$ be the minimizer of $\mathcal{J}(\cdot, \mathbf{u})$. According to $[5$, Theorems 1 and 2]

$$
\beta<\frac{1}{\eta} \Rightarrow b\left(c \beta \eta, \alpha_{1}\right) \leq\|\widehat{\mathbf{f}}-\mathbf{u}\|_{\infty}<b\left(\beta \eta, \alpha_{1}\right)
$$

where

$$
c=\phi^{\prime}\left(\nu_{\mathbf{u}}-2 b\left(\beta \eta, \alpha_{1}\right), \alpha_{2}\right)<1
$$

and $\nu_{\mathbf{u}}$ is a constant computed from the input image. It is worth emphasizing that the upper bound in (13) is independent of the input image $\mathbf{u}$ and of the particular shape of $\phi$ provided that the latter obeys $\mathrm{H} 1-\mathrm{H} 2$. Using our (stronger) assumptions and [5, Lemma 2], for $\beta \eta<1$ fixed, $\alpha_{1} \mapsto b\left(\beta \eta, \alpha_{1}\right)$ is strictly increasing from $(0,+\infty)$ to $(0,+\infty)$. Hence we can choose $\beta \in(0,1 / \eta)$ and $\alpha_{1}>0$ such that the upper bound in (13) equals any fixed number. In our application, we will require that this bound is quite small, around 0.1 , in order to guarantee that $\widehat{\mathbf{f}}$ is close enough to $\mathbf{u}$. According to [5, Theorem 3] and [5, Fig. 7], the number $\nu_{\mathbf{u}}-2 b\left(\beta \eta, \alpha_{1}\right) \gg 0$ is quite large when $b\left(\beta \eta, \alpha_{1}\right)<0.5$. From (14) and $\mathrm{H} 2$, decreasing $\alpha_{2}>0$ towards zero increases $c$ close to one, hence $\|\widehat{\mathbf{f}}-\mathbf{u}\|_{\infty}$ can be arbitrarily close to $b\left(\beta \eta, \alpha_{1}\right)$.

For $\psi(t)=\sqrt{t^{2}+\alpha_{1}}, \phi(t)=\sqrt{t^{2}+\alpha_{2}}$ and $\left|\mathcal{N}_{i}\right|=4=\eta$, we have

$$
\beta<\frac{1}{4} \Rightarrow b\left(\beta \eta, \alpha_{1}\right)=4 \beta \sqrt{\frac{\alpha_{1}}{1-(4 \beta)^{2}}} \text { and } c=\frac{\nu_{\mathbf{u}}-2 b\left(\beta \eta, \alpha_{1}\right)}{\sqrt{\left(\nu_{\mathbf{u}}-2 b\left(\beta \eta, \alpha_{1}\right)\right)^{2}+\alpha_{2}}} .
$$

In our experimental setting, $\beta=0.1$ and $\alpha_{1}=0.05$, which yields $b\left(\beta \eta, \alpha_{1}\right)=0.0976$. For the images in Fig. 5 we computed that $\nu_{\mathbf{u}} \in[52,205]$ in which case choosing $\alpha_{2}=0.05$ entails $c \approx 1$.

\section{NUMERICAL SCHEME}

\section{A. Algorithm}

Given an input image $\mathbf{u}$, the minimizer $\widehat{\mathbf{f}}$ of $\mathcal{J}(\cdot, \mathbf{u})$ provides auxiliary information to sort the pixels of $\mathbf{u}$. More precisely, 2-tuples $(\mathbf{u}[i], \widehat{\mathbf{f}}[i])$ are used as described next

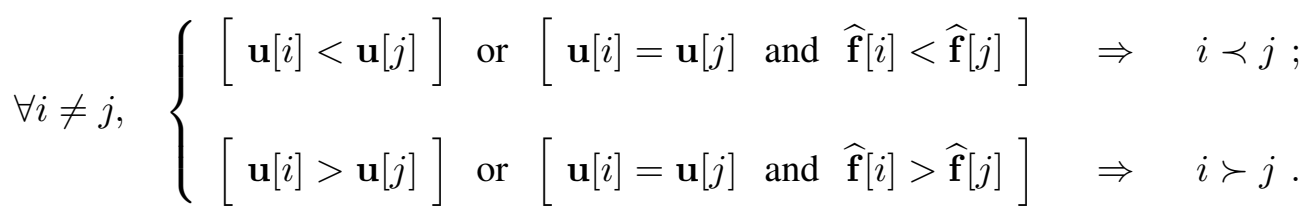

The resulting algorithm for exact histogram specification using the variational approach presented in section III is summarized in Algorithm 1.

Step 2 in the algorithm is aimed to reach a solution $\widehat{\mathbf{f}}$ such that (16) can be applied to all pairs $(i, j), i \neq j$. This algorithm is quite general and can be applied using any functions $(\psi, \phi)$ obeying $\mathrm{H} 1$ and $\mathrm{H} 2$ - for some examples see Table I. 


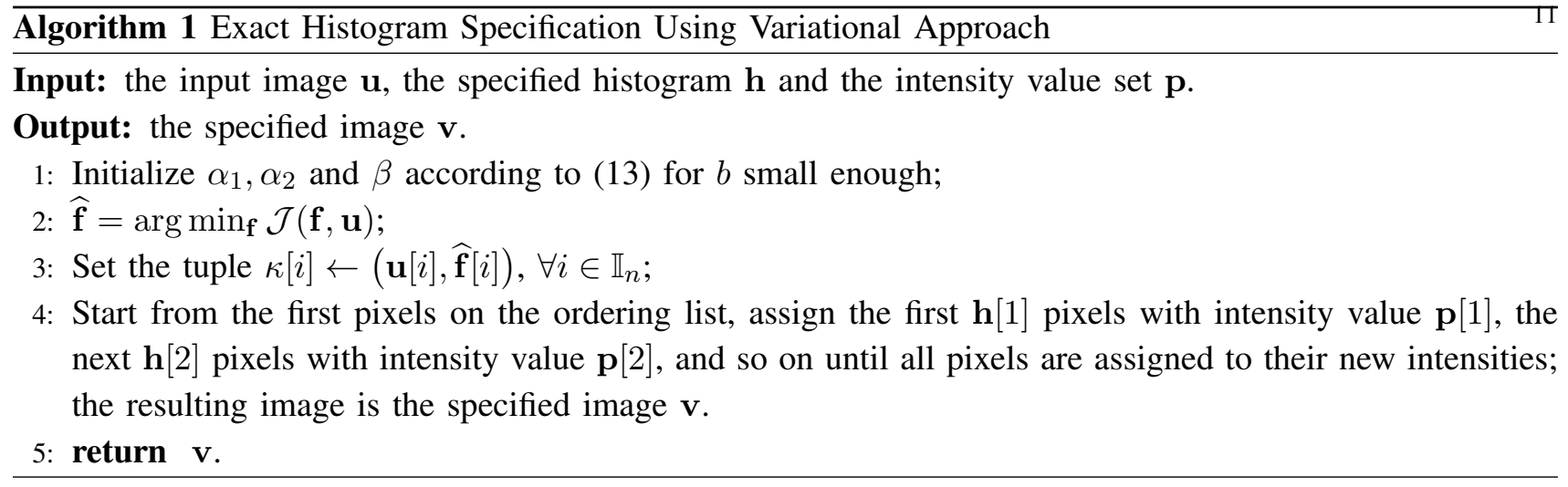

A clear advantage of our method compared with the LM and the WA methods is that in our case ordering is obtained by comparing only two images-the input $\mathbf{u}$ and the minimizer $\widehat{\mathbf{f}}$. The LM method [17] needs to compare (and keep in memory) at least 6 images. For WA method, this number is 9 because the Haar wavelet basis with $2 \times 2$ filters and $4 \times 4$ filters is used.

\section{B. Implementation of Algorithm 1}

There are many algorithms in literature to compute the minimizer $\widehat{\mathbf{f}}$ of $\mathcal{J}(\cdot, \mathbf{u})$ in step 2 of the Algorithm. These include partial differential equation based methods such as explicit method [54], semi-implicit method [36], operator splitting [42], lagged diffusivity fixed point iterations [60], Polak-Ribière CG method [6], majorization-minimization algorithms [7], [24], [31], Nesterov algorithms [40] and so on. We used a pseudoconjugated Polak-Ribière minimization method [6].

For our pixel sorting method, we minimize $\mathcal{J}$ as defined by (3) where $\mathcal{N}_{i}$ is the set of the four adjacent neighbors and

$$
\psi(t)=\sqrt{t^{2}+\alpha_{1}} \text { and } \phi(t)=\sqrt{t^{2}+\alpha_{2}} .
$$

We have $\eta=\left|\mathcal{N}_{i}\right|=4$ hence we must use $\beta<0.25$. Following also the discussion in Remark (a) in section III-A, we select

$$
\beta=0.1 \quad \text { and } \quad \alpha_{1}=\alpha_{2}=0.05 .
$$

According to (15) and (17) we are guaranteed that $\|\widehat{\mathbf{f}}-\mathbf{u}\|_{\infty}<0.0976$, which is a reasonable bound to obtain a real-valued image close enough to $\mathbf{u}$. For the images shown in Fig. 5, the constant $\nu_{\mathbf{u}}$ in (14) ranges between 52 and 205. For our choice of $\alpha_{2}$, the constant $c$ in (13) satisfies $c \in\left(1-10^{-5}, 1\right)$. Therefore, for all these images the minimizer of $\mathcal{J}$ obeys

$$
\|\widehat{\mathbf{f}}-\mathbf{u}\|_{\infty} \lesssim 0.0976
$$

This is a good property ensuring that the minimizer is close enough to the input digital image while the interval is large enough to enable pixels of the minimizer corresponding to each intensity level in $\mathbf{u}$, say $\mathbf{u}[i]=p_{k}$, to take different values in the interval $\left(p_{k}-0.0976, p_{k}+0.0976\right)$.

\section{Some practical limitations}

In spite of the theory presented in section III, it can occur that the minimizer $\widehat{\mathbf{f}}$ of $\mathcal{J}(\cdot, \mathbf{u})$ contains some equally valued pixels. Several important reasons are mentioned next. 
- The real numbers that a computer treats are in fact just a large but finite set of numbers. E.g. Matlab cannot distinguish numbers smaller than $2.2 \times 10^{-16}$.

- The theory supposes that we deal with exact minimizers of $\mathcal{J}$, yet in practice we cannot get such minimizers. Note that a $\mathcal{J}$ satisfying $\mathrm{H} 1$ and $\mathrm{H} 2$ contains large nearly flat regions, so its minimization is not easy. The algorithm being initialized with the digital input image $\mathbf{u}$, an inexact minimizer $\widehat{\mathbf{f}}$ of $\mathcal{J}$ might contain pixels with equal values. Indeed, we have observed that in such cases, increasing the precision of the minimization method usually enables to strictly sort all pixels of $\widehat{\mathbf{f}}$. However, in some cases this could require long computations and for practical reasons, a compromise is done by fixing the maximum number of iterations.

- If the digital image contains large constant regions or if it involves several equal patterns having the same background (this typically can arise for images having a low compression rate), one should expect that the resultant $\widehat{\mathbf{f}}$ can have pixels sharing the same value.

There might be some strategies to improve sorting in case of failure. It is easy to see that the minimizer function $\mathcal{F}: \mathbb{R}^{n} \rightarrow \mathbb{R}^{n}$ is differentiable with respect to all parameters $\alpha_{1}, \alpha_{2}, \beta$. These parameters can slightly be changed so that $\widehat{\mathbf{f}}$ remains close enough to $\mathbf{u}$-rigorous tools for such modifications are provided in subsection III-C. Also, instead of the four adjacent neighbors, on may consider the 8 nearest neighbors. Any such change modifies the possible intersections of $K_{\mathcal{G}}$ and $K_{\mathcal{I}}$ in Theorem 1 with the set $S_{\mathcal{P}}^{n}$ in (11). These questions are not considered here as they would lead to a trickier algorithm.

- Last, we remind that the set $K_{\mathcal{G}} \cup \mathcal{S}_{\mathcal{P}}^{n}$ is not empty.

\section{EXPERIMENTAL RESULTS}

Two practical problems are considered next: contrast compression restoration and equalization inversion using exact histogram specification. Our method to order the pixels was applied as described in (17) and (18). We ran the Polak-Ribière CG minimization with stopping rule given by $\left\|D_{1} \mathcal{J}\left(\widehat{\mathbf{f}}^{k}, \mathbf{u}\right)\right\|_{\infty} \leq n \times 10^{-6}$ and limiting the iteration number to 35. Our method was compared with the local mean (LM) algorithm [17] for $K=6$ and with the wavelet-based algorithm (WA) [61] for Haar wavelet for $K=9$, as recommended by the authors. The experiments were performed using a Mac OS X 10.7.2 and MATLAB v7.12 on a MacBook Air Laptop with an Intel Core i5 1.7 GHZ processor and 4GB of RAM.

Here we present numerical results on 15 digital images of different sizes with gray values in $\{0, \cdots, 255\}$. They are available at http://sipi.usc.edu/database/. We selected images with various sizes, quality and content (presence or quasi-absence of edges, textures, nearly flat regions). The images and their histograms are shown in Fig. 5. We observe that most of these images have quite singular histograms.

In order to measure the results quantitatively, we start out with a given true quantized image w with histogram $\mathbf{h}_{\mathbf{w}}$, we degrade it to obtain an input quantized image $\mathbf{u}$. By applying the three methods on $\mathbf{u}$ with prescribed histogram $\mathbf{h}_{\mathbf{w}}$, we obtain an output image $\mathbf{v}$ which is in fact a restored version of $\mathbf{w}$. We use peak-signal-to-noise-ratio to measure the quality of the output image $\mathbf{v}$ with respect to $\mathbf{w}$, defined as $\mathrm{PSNR}=20 \log _{10}\left(255 N M /\|\mathbf{v}-\mathbf{w}\|_{2}\right)$.

One should keep in mind that

- the performance of LM and WA decrease when the number of gray-values in the input image decreases;

- LM is less efficient for larger image sizes. 


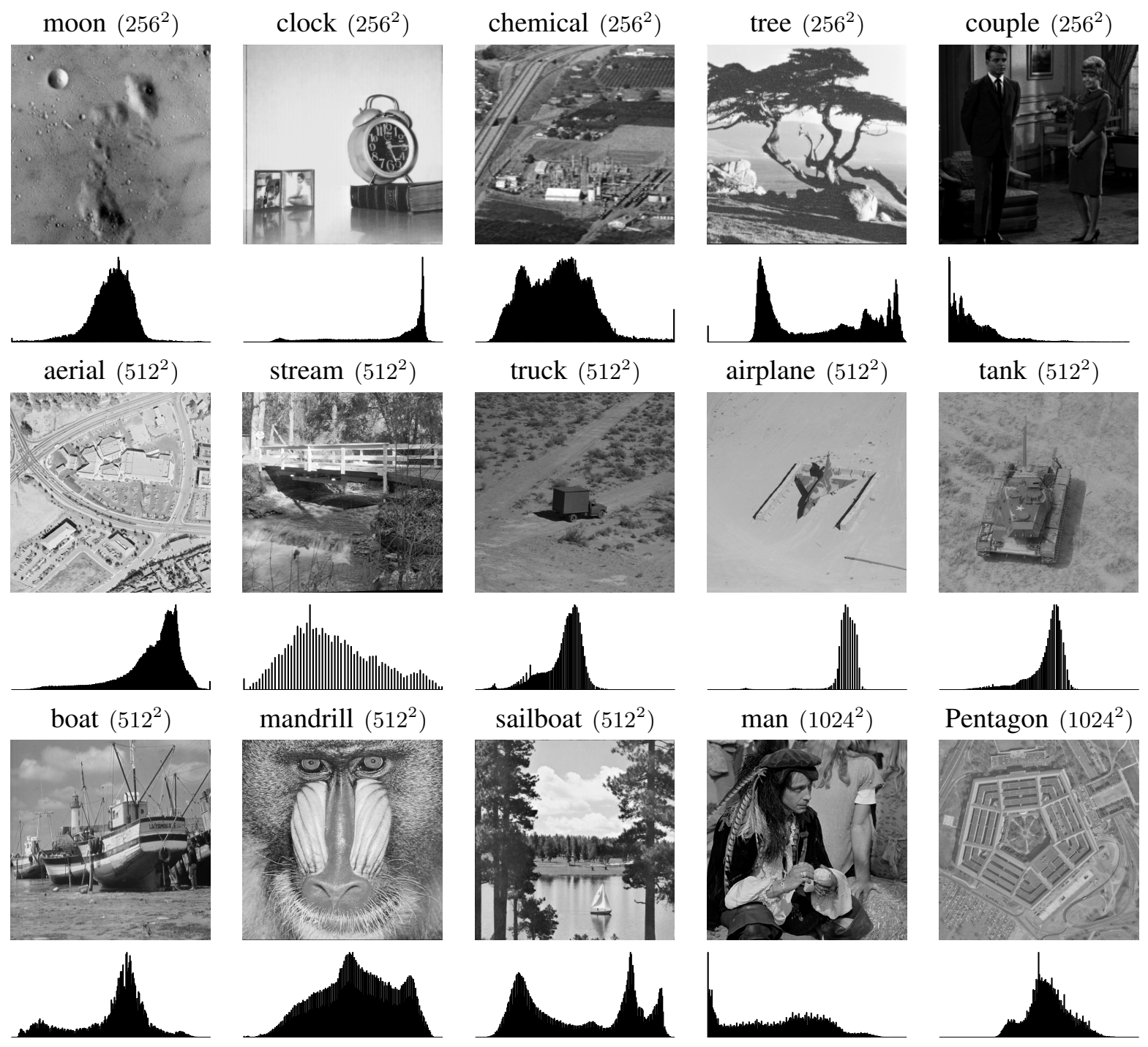

Fig. 5: All 15 digital images used in the tests in this section and their histograms. The gray values of these images belong to $\{0, \cdots, 255\}$.

\section{A. Restoration of Contrast Compression}

The input image $\mathbf{u}$ is obtained from $\mathbf{w}$ by the degradation: $\mathbf{u}=\mathrm{fix}\left(\mathbf{w} / 2^{8-k}\right)$, where $k=1,2, \ldots, 7$. We show the process of contrast compression in Figure 6. This situation arises when a picture is taken with insufficient exposure time, or when we want to compress the image by reducing the number of intensity levels. For example, a 7-bit image can be obtained from an 8-bit image by using $k=7$. In the tests, we used LM, WA and our method to obtain the output images $\mathbf{v}$ having a prescribed histogram $\mathbf{h}_{\mathbf{w}}$.

We compare LM, WA and our algorithm. The comparison results are shown in Table II-IV for 3, 4, 5 bits per pixel, respectively, where the best results are printed in boldface. We see from the PSNR values that our method outperforms LM and WA in quality in all cases; this can be explained by the constraint that $\|\widehat{\mathbf{f}}-\mathbf{u}\|_{\infty}<0.1$ and that our algorithm enables a strongly better strict ordering.

One important indicator for a good exact histogram specification algorithm is to observe if it can establish a strict ordering for all the pixels. If a sorting method yields two or more pixels sharing the same value 


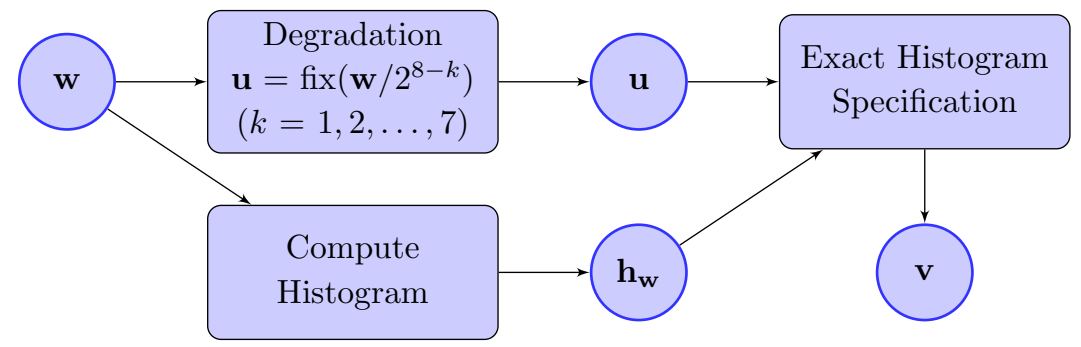

Fig. 6: Contrast Compression.

we call the pixels failure pixels. Table II-IV shows the percentages of failure pixels produced by the three methods. We find that LM and WA have a high number of equally valued pixels.

TABLE II: Data are 3-bits images. The PSNR (dB) between the true image $\mathbf{w}$ and the output image $\mathbf{v}$.

\begin{tabular}{c|ccc|ccc|ccc} 
& \multicolumn{3}{|c}{ PSNR } & \multicolumn{3}{c|}{ Fail $\%$} & \multicolumn{3}{c}{ CPU } \\
\hline Image & LM & WA & Ours & LM & WA & Ours & LM & WA & Ours \\
\hline moon & 31.15 & 30.70 & $\mathbf{3 1 . 6 8}$ & 52.29 & 75.75 & $\mathbf{0 . 0 4}$ & $\mathbf{0 . 4 4}$ & 0.96 & 1.08 \\
clock & 31.32 & 30.68 & $\mathbf{3 2 . 6 0}$ & 67.93 & 76.58 & $\mathbf{3 0 . 1 2}$ & $\mathbf{0 . 3 7}$ & 0.74 & 0.74 \\
chemical & 30.20 & 29.24 & $\mathbf{3 0 . 4 7}$ & 34.28 & 55.97 & $\mathbf{0 . 0 4}$ & $\mathbf{0 . 4 0}$ & 0.77 & 1.08 \\
tree & 29.86 & 29.20 & $\mathbf{3 0 . 3 8}$ & 46.45 & 59.63 & $\mathbf{6 . 1 5}$ & $\mathbf{0 . 4 3}$ & 0.82 & 1.02 \\
couple & 29.30 & 28.77 & $\mathbf{2 9 . 7 4}$ & 75.90 & 82.72 & $\mathbf{1 2 . 8 3}$ & $\mathbf{0 . 3 4}$ & 0.77 & 1.03 \\
aerial & 29.39 & 28.62 & $\mathbf{2 9 . 7 4}$ & 57.83 & 67.72 & $\mathbf{3 . 1 9}$ & $\mathbf{1 . 6 8}$ & 4.49 & 4.19 \\
stream & 29.22 & 28.60 & $\mathbf{2 9 . 4 6}$ & 34.68 & 57.85 & $\mathbf{0 . 0 6}$ & $\mathbf{1 . 7 8}$ & 4.32 & 3.68 \\
truck & 32.03 & 31.34 & $\mathbf{3 2 . 3 6}$ & 69.49 & 89.60 & $\mathbf{2 . 3 3}$ & $\mathbf{1 . 7 0}$ & 4.55 & 3.90 \\
airplane & 31.16 & 30.76 & $\mathbf{3 2 . 8 6}$ & 88.27 & 94.37 & $\mathbf{4 1 . 2 0}$ & $\mathbf{1 . 3 6}$ & 4.37 & 3.88 \\
tank & 31.27 & 30.74 & $\mathbf{3 1 . 6 7}$ & 75.13 & 89.74 & $\mathbf{0 . 6 0}$ & $\mathbf{1 . 7 9}$ & 4.71 & 3.97 \\
boat & 30.59 & 29.75 & $\mathbf{3 1 . 1 8}$ & 63.46 & 78.81 & $\mathbf{7 . 7 6}$ & $\mathbf{1 . 6 7}$ & 4.33 & 3.65 \\
mandrill & 28.65 & 28.21 & $\mathbf{2 8 . 9 5}$ & 27.13 & 46.73 & $\mathbf{0 . 1 0}$ & $\mathbf{1 . 7 5}$ & 4.60 & 3.97 \\
sailboat & 30.72 & 29.74 & $\mathbf{3 1 . 3 4}$ & 56.14 & 71.63 & $\mathbf{6 . 4 0}$ & $\mathbf{1 . 6 3}$ & 4.10 & 3.48 \\
man & 31.27 & 30.42 & $\mathbf{3 1 . 6 2}$ & 75.60 & 87.92 & $\mathbf{6 . 8 9}$ & $\mathbf{8 . 2 7}$ & 20.81 & 12.70 \\
Pentagon & 30.33 & 29.84 & $\mathbf{3 0 . 6 6}$ & 68.82 & 87.35 & $\mathbf{0 . 5 8}$ & $\mathbf{9 . 5 6}$ & 23.52 & 13.66 \\
\hline means & 30.43 & 29.77 & $\mathbf{3 0 . 9 8}$ & 59.56 & 74.83 & $\mathbf{7 . 8 9}$ & $\mathbf{2 . 2 1}$ & 5.59 & 4.14
\end{tabular}

\section{B. Histogram Equalization Inversion}

We consider the application of the histogram equalization inversion, which is to recover the true quantized image $\mathbf{w}$ with histogram $\mathbf{h}_{\mathbf{w}}$ from its specified version $\mathbf{u}$. This was the main application used in [17] to demonstrate the interest of the LM method. Let $\mathbf{u}=T(\mathbf{w}, \mathbf{h})$ be the process to specify the image w with histogram $\mathbf{h}_{\mathbf{w}}$ to an image $\mathbf{u}$ such that the histogram of $\mathbf{u}$ is $\mathbf{h}$. The quantized image $\mathbf{w}$ can be exactly recovered by $T\left(\mathbf{u}, \mathbf{h}_{\mathbf{w}}\right)$ under the hypothesis of order preservation by the method. Since the ordering among the pixels of $\mathbf{w}$ is not identical with that among pixels of $\mathbf{u}$, the recovered image $\mathbf{v}=T\left(\mathbf{u}, \mathbf{h}_{\mathbf{w}}\right)$ is just an approximation of $\mathbf{w}$. We show the process of histogram equalization inversion in Figure 7.

Table V shows the PSNR, the failure percentage, the CPU running time of the results by the three methods. We notice from Table V that WA method yields better PSNR than LM method in all images. In all these cases, our results are much better. Whereas our method can sort all the pixels in all the 8-bit images tested, the only 8-bit image where the other two methods successfully sort is the "mandrill" image. All these results show that the ordering enabled by our method better fits real-world digital images. 
TABLE III: Data are 4-bits images. The PSNR $(\mathrm{dB})$ between the true image $\mathbf{w}$ and the output image $\mathbf{v}{ }^{15}$

\begin{tabular}{c|ccc|ccc|ccc} 
& \multicolumn{3}{|c}{} & PSNR & \multicolumn{3}{c|}{ Fail $\%$} & \multicolumn{3}{c}{ CPU } \\
\hline Image & LM & WA & Ours & LM & WA & Ours & LM & WA & Ours \\
\hline moon & 35.25 & 34.74 & $\mathbf{3 5 . 5 1}$ & 16.75 & 40.14 & $\mathbf{0 . 0 0}$ & $\mathbf{0 . 4 7}$ & 1.20 & 0.95 \\
clock & 36.14 & 35.66 & $\mathbf{3 7 . 5 2}$ & 52.54 & 62.66 & $\mathbf{1 8 . 1 7}$ & $\mathbf{0 . 3 2}$ & 0.77 & 1.15 \\
chemical & 34.71 & 33.83 & $\mathbf{3 5 . 0 5}$ & 8.70 & 20.01 & $\mathbf{0 . 0 1}$ & $\mathbf{0 . 4 1}$ & 0.81 & 1.00 \\
tree & 34.76 & 34.34 & $\mathbf{3 4 . 9 5}$ & 22.15 & 36.02 & $\mathbf{4 . 0 5}$ & $\mathbf{0 . 3 4}$ & 0.72 & 1.13 \\
couple & 35.11 & 34.43 & $\mathbf{3 5 . 6 3}$ & 49.56 & 60.04 & $\mathbf{6 . 9 6}$ & $\mathbf{0 . 4 4}$ & 0.73 & 0.89 \\
aerial & 34.46 & 33.76 & $\mathbf{3 4 . 9 5}$ & 28.56 & 42.21 & $\mathbf{0 . 3 6}$ & $\mathbf{1 . 7 9}$ & 4.38 & 3.87 \\
stream & 34.18 & 33.73 & $\mathbf{3 4 . 4 9}$ & 9.61 & 20.25 & $\mathbf{0 . 0 0}$ & $\mathbf{1 . 7 6}$ & 5.62 & 4.12 \\
truck & 35.93 & 35.23 & $\mathbf{3 6 . 1 9}$ & 39.81 & 60.85 & $\mathbf{0 . 5 0}$ & $\mathbf{1 . 9 2}$ & 5.92 & 3.50 \\
airplane & 37.72 & 37.28 & $\mathbf{3 8 . 7 0}$ & 75.92 & 88.18 & $\mathbf{7 . 8 8}$ & $\mathbf{1 . 9 9}$ & 4.64 & 3.99 \\
tank & 35.49 & 34.90 & $\mathbf{3 5 . 6 9}$ & 44.58 & 64.84 & $\mathbf{0 . 0 1}$ & $\mathbf{2 . 0 4}$ & 5.63 & 4.03 \\
boat & 35.67 & 34.77 & $\mathbf{3 6 . 1 3}$ & 32.86 & 52.41 & $\mathbf{0 . 2 9}$ & $\mathbf{2 . 0 6}$ & 5.18 & 4.22 \\
mandrill & 33.40 & 33.12 & $\mathbf{3 3 . 7 5}$ & 8.15 & 17.16 & $\mathbf{0 . 0 3}$ & $\mathbf{1 . 8 8}$ & 4.80 & 4.17 \\
sailboat & 35.29 & 34.65 & $\mathbf{3 5 . 5 2}$ & 27.94 & 46.96 & $\mathbf{0 . 5 1}$ & $\mathbf{1 . 7 6}$ & 4.70 & 4.33 \\
man & 35.78 & 34.94 & $\mathbf{3 6 . 1 3}$ & 46.59 & 64.64 & $\mathbf{3 . 4 3}$ & $\mathbf{8 . 8 4}$ & 24.76 & 11.91 \\
Pentagon & 34.71 & 34.32 & $\mathbf{3 5 . 1 6}$ & 37.84 & 61.45 & $\mathbf{0 . 0 5}$ & $\mathbf{1 0 . 0 4}$ & 26.14 & 12.33 \\
\hline means & 34.73 & 34.07 & $\mathbf{3 5 . 2 0}$ & 32.80 & 48.80 & $\mathbf{2 . 5 3}$ & $\mathbf{2 . 3 3}$ & 6.53 & 4.07
\end{tabular}

TABLE IV: Data are 5-bits images. The PSNR (dB) between the true image $\mathbf{w}$ and the output image $\mathbf{v}$.

\begin{tabular}{c|ccc|ccc|ccc} 
& \multicolumn{3}{|c}{} & PSNR & \multicolumn{3}{c|}{ Fail $\%$} & \multicolumn{3}{c}{ CPU } \\
\hline Image & LM & WA & Ours & LM & WA & Ours & LM & WA & Ours \\
\hline moon & 39.69 & 39.37 & $\mathbf{3 9 . 8 1}$ & 0.62 & 3.44 & $\mathbf{0 . 0 0}$ & $\mathbf{0 . 4 7}$ & 0.98 & 1.16 \\
clock & 41.24 & 40.79 & $\mathbf{4 2 . 2 9}$ & 39.10 & 48.76 & $\mathbf{8 . 2 5}$ & $\mathbf{0 . 4 0}$ & 0.75 & 0.83 \\
chemical & 39.52 & 38.95 & $\mathbf{3 9 . 7 8}$ & 0.72 & 2.21 & $\mathbf{0 . 0 0}$ & $\mathbf{0 . 4 9}$ & 0.76 & 1.14 \\
tree & 39.78 & 39.51 & $\mathbf{4 0 . 1 7}$ & 13.40 & 18.34 & $\mathbf{1 . 1 6}$ & $\mathbf{0 . 3 3}$ & 0.77 & 0.93 \\
couple & 40.56 & 39.85 & $\mathbf{4 1 . 1 7}$ & 24.82 & 33.59 & $\mathbf{0 . 8 0}$ & $\mathbf{0 . 4 1}$ & 1.22 & 1.00 \\
aerial & 39.70 & 39.19 & $\mathbf{4 0 . 2 0}$ & 12.38 & 19.05 & $\mathbf{0 . 0 8}$ & $\mathbf{1 . 8 5}$ & 4.35 & 4.67 \\
stream & 39.99 & 39.72 & $\mathbf{4 0 . 1 4}$ & 1.82 & 4.16 & $\mathbf{0 . 0 0}$ & $\mathbf{1 . 7 1}$ & 4.30 & 4.05 \\
truck & 40.31 & 39.77 & $\mathbf{4 0 . 5 2}$ & 8.03 & 22.80 & $\mathbf{0 . 0 0}$ & $\mathbf{2 . 2 0}$ & 4.75 & 3.87 \\
airplane & 41.97 & 41.75 & $\mathbf{4 2 . 1 6}$ & 60.54 & 77.30 & $\mathbf{0 . 2 6}$ & $\mathbf{1 . 8 3}$ & 4.41 & 3.75 \\
tank & 39.89 & 39.45 & $\mathbf{4 0 . 0 3}$ & 8.41 & 16.95 & $\mathbf{0 . 0 0}$ & $\mathbf{1 . 9 6}$ & 4.55 & 3.94 \\
boat & 39.86 & 39.34 & $\mathbf{4 0 . 1 1}$ & 7.11 & 19.02 & $\mathbf{0 . 0 5}$ & $\mathbf{1 . 6 2}$ & 4.32 & 3.96 \\
mandrill & 38.71 & 38.52 & $\mathbf{3 8 . 9 3}$ & 0.36 & 1.91 & $\mathbf{0 . 0 0}$ & $\mathbf{1 . 6 0}$ & 3.75 & 8.69 \\
sailboat & 39.74 & 39.45 & $\mathbf{3 9 . 8 8}$ & 9.94 & 19.91 & $\mathbf{0 . 0 3}$ & $\mathbf{1 . 6 4}$ & 4.30 & 8.76 \\
man & 40.24 & 39.68 & $\mathbf{4 0 . 4 4}$ & 15.38 & 26.30 & $\mathbf{0 . 1 8}$ & $\mathbf{8 . 2 5}$ & 20.98 & 12.60 \\
Pentagon & 39.30 & 39.07 & $\mathbf{3 9 . 5 6}$ & 5.47 & 20.11 & $\mathbf{0 . 0 1}$ & $\mathbf{8 . 5 1}$ & 21.64 & 11.84 \\
\hline means & 39.40 & 39.07 & $\mathbf{3 9 . 9 3}$ & 13.40 & 21.80 & $\mathbf{0 . 6 0}$ & $\mathbf{2 . 2 0}$ & 5.47 & 4.87
\end{tabular}

Figures 9 gives the enlarged portions of the difference images on "Aerial", "Couple", "Man" images, the corresponding enlarged portions of the input image are shown in Figure 8. We can discern more features in the difference images by both LM method and WA method than by our method. It demonstrates that our algorithm yields the best restoration.

\section{CONCLUSIONS AND PERSPECTIVES}

In this paper, we propose a variational approach for exact histogram specification. Since the energy that we minimize is smooth, its minimizers are real-valued images whose pixels are different from each other with a very high probability. The latter enables us to strictly order the pixels of generically all 8-bit images and to obtain much better ordering results than the competing methods in all cases. Our parameter choice guarantees that $\|\widehat{\mathbf{f}}-\mathbf{u}\|_{\infty} \lesssim 0.1$ and the choice of $\mathcal{J}$ is such that this small residual contains some quantization noise. 


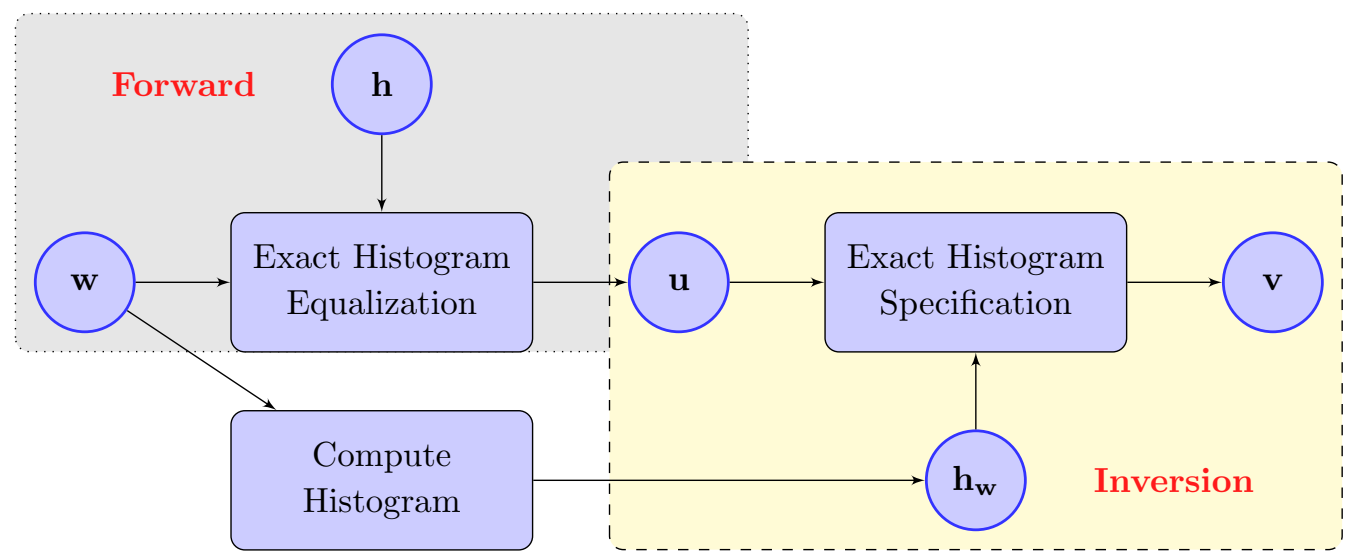

Fig. 7: Histogram Equalization Inversion. A true quantized image $\mathbf{w}$ with histogram $\mathbf{h}_{\mathbf{w}}$ is equalized to the input image $\mathbf{u}$ using exact histogram specification method. The task is to restore $\mathbf{w}$ from the input image $\mathbf{u}$ and the prescribed histogram $\mathbf{h}_{\mathbf{w}}$ using the same exact histogram specification method. The specified image $\mathbf{v}$ is a restored version of $\mathbf{w}$.

TABLE V: The PSNR (dB) between the true image $\mathbf{w}$ and output images $\mathbf{v}$.

\begin{tabular}{c|ccc|ccc|ccc} 
& \multicolumn{3}{|c|}{ PSNR } & \multicolumn{3}{c|}{ Fail $\%$} & \multicolumn{3}{c}{ CPU } \\
\hline Image & LM & WA & Ours & LM & WA & Ours & LM & WA & Ours \\
\hline moon & 47.39 & 46.50 & $\mathbf{4 7 . 8 3}$ & 0.07 & 0.11 & $\mathbf{0 . 0 0}$ & $\mathbf{0 . 6 7}$ & 1.10 & 3.58 \\
clock & 51.69 & 51.56 & $\mathbf{5 1 . 7 9}$ & 0.53 & 2.41 & $\mathbf{0 . 0 0}$ & $\mathbf{0 . 6 5}$ & 1.20 & 2.01 \\
chemical & 49.34 & 48.90 & $\mathbf{4 9 . 6 7}$ & 0.03 & 0.10 & $\mathbf{0 . 0 0}$ & $\mathbf{0 . 6 7}$ & 1.06 & 3.05 \\
tree & 51.94 & 51.84 & $\mathbf{5 2 . 0 2}$ & 0.03 & 0.18 & $\mathbf{0 . 0 0}$ & $\mathbf{0 . 5 4}$ & 1.12 & 2.81 \\
couple & 44.23 & 43.71 & $\mathbf{4 4 . 6 6}$ & 1.38 & 1.97 & $\mathbf{0 . 0 0}$ & $\mathbf{0 . 6 0}$ & 1.13 & 2.35 \\
aerial & 48.36 & 48.06 & $\mathbf{5 0 . 0 6}$ & 0.00 & 0.02 & $\mathbf{0 . 0 0}$ & $\mathbf{2 . 8 6}$ & 6.08 & 9.35 \\
stream & 44.76 & 45.00 & $\mathbf{4 5 . 0 8}$ & 0.37 & 0.71 & $\mathbf{0 . 0 0}$ & $\mathbf{2 . 6 5}$ & 6.13 & 7.98 \\
truck & 49.67 & 49.51 & $\mathbf{4 9 . 9 6}$ & 0.12 & 0.24 & $\mathbf{0 . 0 0}$ & $\mathbf{2 . 8 2}$ & 5.90 & 12.43 \\
airplane & 46.74 & 46.26 & $\mathbf{4 7 . 2 4}$ & 1.73 & 8.96 & $\mathbf{0 . 0 0}$ & $\mathbf{3 . 0 1}$ & 7.40 & 8.40 \\
tank & 48.11 & 48.15 & $\mathbf{4 8 . 3 4}$ & 0.02 & 0.06 & $\mathbf{0 . 0 0}$ & $\mathbf{2 . 9 5}$ & 6.70 & 12.53 \\
boat & 49.51 & 49.58 & $\mathbf{4 9 . 8 9}$ & 0.06 & 0.09 & $\mathbf{0 . 0 0}$ & $\mathbf{2 . 8 8}$ & 5.97 & 13.08 \\
mandrill & 48.27 & 49.47 & $\mathbf{4 9 . 7 6}$ & 0.00 & 0.00 & $\mathbf{0 . 0 0}$ & $\mathbf{2 . 5 6}$ & 5.33 & 12.68 \\
sailboat & 50.85 & 50.72 & $\mathbf{5 1 . 3 0}$ & 0.01 & 0.05 & $\mathbf{0 . 0 0}$ & $\mathbf{2 . 5 8}$ & 5.29 & 17.16 \\
man & 49.23 & 49.18 & $\mathbf{4 9 . 4 4}$ & 0.17 & 0.40 & $\mathbf{0 . 0 0}$ & $\mathbf{1 3 . 0 3}$ & 28.01 & 39.96 \\
Pentagon & 50.69 & 50.62 & $\mathbf{5 1 . 3 6}$ & 0.01 & 0.01 & $\mathbf{0 . 0 0}$ & $\mathbf{1 3 . 7 4}$ & 29.32 & 39.48 \\
\hline means & 48.72 & 48.60 & $\mathbf{4 9 . 2 3}$ & 0.30 & 1.02 & $\mathbf{0 . 0 0}$ & $\mathbf{3 . 4 8}$ & 7.45 & 12.46
\end{tabular}

This, combined with the much better ordering rate, explains why the obtained results outperform the existing methods. In all tested cases, our method gave not only a much better ordering, but also yielded the best PSNR. Hence the ordering provided by our method fits better all these various real-world digital images.

Our method is simple and obviously very efficient though we used a standard minimization algorithm. We envisage the conception of a specialized minimization scheme in order to improve the computation speed. Extension to color images will also be considered.

\section{APPENDIX}

Given a square matrix $A$, the expression $A \succ 0$ means that $A$ is positive definite and $A \succeq 0$ that $A$ is positive semi-definite. 

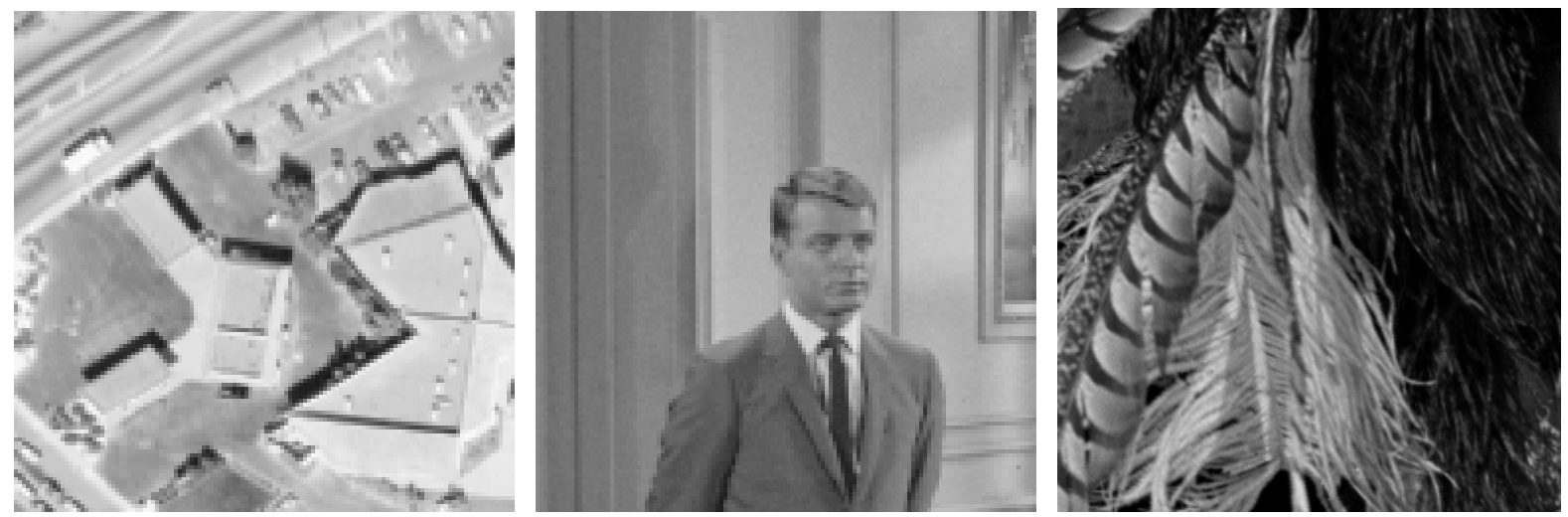

Fig. 8: Enlarged portions of "Aerial" image, "Couple" image, and "Man" image, respectively.

\section{A. Proof of Proposition 1}

By $\mathrm{H} 1$, for any $\mathbf{u} \in \mathbb{R}^{n}$, the function $\mathcal{J}(\cdot, \mathbf{u})$ in (2) is strictly convex and coercive, hence for any $\mathbf{u}$ and $\beta>0$, it has a unique minimizer. Each minimizer point $\widehat{\mathbf{f}}$ of $\mathcal{J}(\widehat{\mathbf{f}}, \mathbf{u})$ is determined by $D_{1} \mathcal{J}(\widehat{\mathbf{f}}, \mathbf{u})=0$. We have

$$
0=D_{1} \mathcal{J}(\widehat{\mathbf{f}}, \mathbf{u})=D_{1} \Psi(\widehat{\mathbf{f}}, \mathbf{u})+\beta D_{1} \Phi(\widehat{\mathbf{f}})
$$

where

$$
D_{1} \Psi(\widehat{\mathbf{f}}, \mathbf{u})=\left[\begin{array}{c}
\psi^{\prime}(\widehat{\mathbf{f}}[1]-\mathbf{u}[1]) \\
\cdots \\
\psi^{\prime}(\widehat{\mathbf{f}}[p]-\mathbf{u}[p])
\end{array}\right]^{T} \quad \text { and } \quad D_{1} \Phi(\widehat{\mathbf{f}})=\left[\begin{array}{c}
\phi^{\prime}\left(\mathbf{g}_{1}^{T} \widehat{\mathbf{f}}\right) \\
\ldots \\
\phi^{\prime}\left(\mathbf{g}_{r}^{T} \widehat{\mathbf{f}}\right)
\end{array}\right]^{T} G
$$

Differentiation with respect to $\widehat{\mathbf{f}}$ yet again yields

$$
D_{1}^{2} \mathcal{J}(\widehat{\mathbf{f}}, \mathbf{u})=D_{1}^{2} \Psi(\widehat{\mathbf{f}}, \mathbf{u})+\beta D_{1}^{2} \Phi(\widehat{\mathbf{f}}) \in \mathbb{R}^{n \times n} .
$$

Here, $D_{1}^{2} \Psi(\widehat{\mathbf{f}}, \mathbf{u})$ is an $n \times n$ diagonal matrix with strictly positive entries according to $\mathrm{H} 1$ :

$$
D_{1}^{2} \Psi(\widehat{\mathbf{f}}, \mathbf{u})[i, i]=\psi^{\prime \prime}(\widehat{\mathbf{f}}[i]-\mathbf{u}[i]), \quad \forall i \in \mathbb{I}_{n} .
$$

Hence $D_{1}^{2} \Psi(\mathcal{F}(\mathbf{u}), \mathbf{u}) \succ 0$. Furthermore,

$$
D_{1}^{2} \Phi(\widehat{\mathbf{f}})=G^{T} \operatorname{diag}\left(\phi^{\prime \prime}\left(\mathbf{g}_{1}^{T} \widehat{\mathbf{f}}\right), \cdots, \phi^{\prime \prime}\left(\mathbf{g}_{r}^{T} \widehat{\mathbf{f}}\right)\right) G,
$$

so $D_{1}^{2} \Phi(\widehat{\mathbf{f}}) \succeq 0$. It follows that $D_{1}^{2} \mathcal{J}(\widehat{\mathbf{f}}, \mathbf{u}) \succ 0$, for any $\mathbf{u} \in \mathbb{R}^{n}$.

Consequently, Lemma 1 holds true for any $\mathbf{u} \in \mathbb{R}^{n}$ and any $\beta>0$. The same lemma shows that the statement of Proposition 1 holds true for $\mathcal{O}=\mathbb{R}^{n}$.

\section{B. Proof of Lemma 2}

Since $\mathcal{F}$ is a local minimizer function,

$$
D_{1} \mathcal{J}(\mathcal{F}(\mathbf{u}), \mathbf{u})=0, \quad \forall \mathbf{u} \in \mathbb{R}^{n} .
$$

We can thus differentiate with respect to $\mathbf{u}$ on both sides of (24) and yields

$$
D_{1}^{2} \mathcal{J}(\mathcal{F}(\mathbf{u}), \mathbf{u}) D \mathcal{F}(\mathbf{u})+D_{2} D_{1} \mathcal{J}(\mathcal{F}(\mathbf{u}), \mathbf{u})=0, \quad \forall \mathbf{u} \in \mathbb{R}^{n}
$$



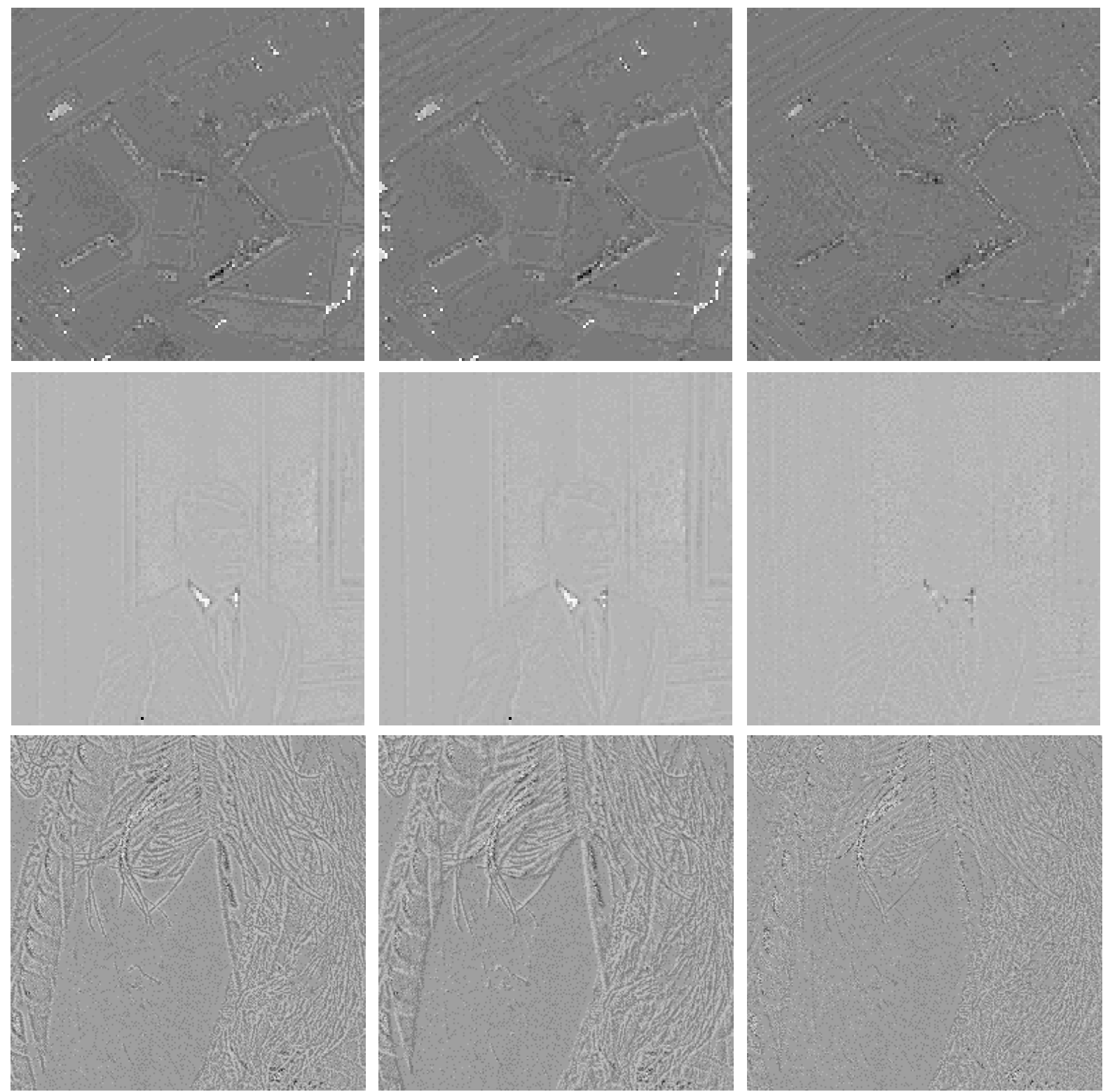

Fig. 9: The enlarged portions of the different images between $\mathbf{w}$ and $\mathbf{v}$ by LM (Left), WA (Middle) and our method (Right) for "Aerial" image (first row), "Couple" image (second row), and "Man" image (third row). Our method yields fewest features in the difference images.

Note that $D \mathcal{F}(\mathbf{u})$ and $D_{2} D_{1} \mathcal{J}(\mathcal{F}(\mathbf{u}), \mathbf{u})$ are $n \times n$ real matrices. The Hessian matrix $H(\mathbf{u})=D_{1}^{2} \mathcal{J}(\mathcal{F}(\mathbf{u}), \mathbf{u})$ can be expanded using (21):

$$
H(\mathbf{u})=D_{1}^{2} \Psi(\mathcal{F}(\mathbf{u}), \mathbf{u})+\beta D_{1}^{2} \Phi(\mathcal{F}(\mathbf{u})) \in \mathbb{R}^{n \times n} .
$$

Replacing $\widehat{\mathbf{f}}$ by $\mathcal{F}(\mathbf{u})$ in (22) and (23) yields

$$
H(\mathbf{u})=\operatorname{diag}\left(\left\{\psi^{\prime \prime}\left(\mathcal{F}_{i}(\mathbf{u})-\mathbf{u}[i]\right)\right\}_{i=1}^{n}\right)+\beta G^{T} \operatorname{diag}\left(\left\{\phi^{\prime \prime}\left(\mathbf{g}_{i}^{T} \mathcal{F}(\mathbf{u})\right)\right\}_{i=1}^{n}\right) G,
$$

as stated in (5). Using $\mathrm{H} 1$, it is readily seen that

$$
\operatorname{diag}\left(\left\{\psi^{\prime \prime}\left(\mathcal{F}_{i}(\mathbf{u})-\mathbf{u}[i]\right)\right\}_{i=1}^{n}\right) \succ 0 \quad \text { and } \quad G^{T} \operatorname{diag}\left(\left\{\phi^{\prime \prime}\left(\mathbf{g}_{i}^{T} \mathcal{F}(\mathbf{u})\right)\right\}_{i=1}^{n}\right) G \succeq 0 .
$$

Then $H(\mathbf{u}) \succ 0$, hence $H(\mathbf{u})$ is invertible. 
Using (19) and (20), where we consider $\mathcal{F}(\mathbf{u})$ in place of $\widehat{\mathbf{f}}$, shows that

$$
D_{2} D_{1} \mathcal{J}(\mathcal{F}(\mathbf{u}), \mathbf{u})=D_{2} D_{1} \Psi(\mathcal{F}(\mathbf{u}), \mathbf{u})=-\operatorname{diag}\left(\left\{\psi^{\prime \prime}\left(\mathcal{F}_{i}(\mathbf{u})-\mathbf{u}[i]\right)\right\}_{i=1}^{n}\right) .
$$

Then (25) is equivalent to

$$
D \mathcal{F}(\mathbf{u})=-(H(\mathbf{u}))^{-1} D_{2} D_{1} \mathcal{J}(\mathcal{F}(\mathbf{u}), \mathbf{u})=(H(\mathbf{u}))^{-1} \operatorname{diag}\left(\left\{\psi^{\prime \prime}\left(\mathcal{F}_{i}(\mathbf{u})-\mathbf{u}[i]\right)\right\}_{i=1}^{n}\right), \quad \forall \mathbf{u} \in \mathbb{R}^{n} .
$$

Obviously, $\operatorname{rank}(D \mathcal{F}(\mathbf{u}))=n$. This result is independent of the value of $\beta>0$.

\section{Proof of Theorem 1}

The proof consists of two parts.

(I) Given $g \in \mathcal{G}$, where $\mathcal{G}$ is given in (8), consider the function $f_{g}: \mathbb{R}^{n} \rightarrow \mathbb{R}$ defined by

$$
f_{g}(\mathbf{u})=g^{T} \mathcal{F}(\mathbf{u}), \quad \forall \mathbf{u} \in \mathbb{R}^{n} .
$$

By Lemma 2, $D \mathcal{F}(\mathbf{u})$ is invertible. Hence ${ }^{5} g^{T} D \mathcal{F}(\mathbf{u}) \neq 0$ and thus

$$
\operatorname{rank}\left(f_{g}(\mathbf{u})\right)=\operatorname{rank}\left(g^{T} D \mathcal{F}(\mathbf{u})\right)=1, \quad \forall \mathbf{u} \in \mathbb{R}^{n} .
$$

In particular, $f_{g}$ does not have critical points. Its inverse of the origin $K_{g}$ reads

$$
K_{g} \stackrel{\text { def }}{=} f_{g}^{-1}(0)=\left\{\mathbf{u} \in \mathbb{R}^{n} \mid g^{T} \mathcal{F}(\mathbf{u})=0\right\} .
$$

By an extension ${ }^{6}$ of the constant rank theorem [4], the subset $K_{g}$ in (28), supposed nonempty, is a $\mathcal{C}^{s-1}$ manifold of $\mathbb{R}^{n}$ of dimension $n-1$. Hence $\mathbb{L}^{n}\left(K_{g}\right)=0$ (see e.g. [21], [35]). Using Proposition $1, f_{g}$ is $\mathcal{C}^{s-1}$-continuous, so $K_{g}$ is closed.

The set $K_{\mathcal{G}}$ in (9) also reads

$$
K_{\mathcal{G}}=\bigcup_{g \in \mathcal{G}} K_{g}
$$

Using that $\mathcal{G}$ is of finite cardinality, it follows that $K_{\mathcal{G}}$ is closed in $\mathbb{R}^{n}$ and that

$$
\mathbb{L}^{n}\left(K_{\mathcal{G}}\right)=0 .
$$

The conclusion is clearly independent of the value of $\beta>0$.

(II) Given $(i, j) \in \mathbb{I}_{n} \times \mathbb{I}_{n}$ (including $i=j$ ), define the subset $K_{i, j} \subset \mathbb{R}^{n}$ as

$$
K_{i, j}=\mathcal{F}_{i}^{-1}\left(\mathbf{u}_{j}\right)=\left\{\mathbf{u} \in \mathbb{R}^{n} \mid \mathcal{F}_{i}(\mathbf{u})=\mathbf{u}_{j}\right\} .
$$

From Proposition $1, \mathcal{F}_{i}: \mathbb{R}^{n} \rightarrow \mathbb{R}$ is $\mathcal{C}^{s-1}$ continuous, so $K_{i, j}$ is closed in $\mathbb{R}^{n}$. By Lemma 2 , all rows of $D \mathcal{F}(\mathbf{u}) \in \mathbb{R}^{n \times n}$ are linearly independent, $\forall \mathbf{u} \in \mathbb{R}^{n}$. Consequently, for any $i \in \mathbb{I}_{n}$,

$$
\operatorname{rank}\left(D \mathcal{F}_{i}(\mathbf{u})\right)=1, \quad \forall \mathbf{u} \in \mathbb{R}^{n}
$$

Using the same arguments as in (I), $K_{i, j}$ is a $\mathcal{C}^{s-1}$ submanifold of $\mathbb{R}^{n}$ of dimension $n-1$, hence

$$
\mathbb{L}^{n}\left(K_{i, j}\right)=0 \text {. }
$$

Noticing that $K_{\mathcal{I}}$ in (10) is a finite union of $(n-1)$-dimensional submanifolds in $\mathbb{R}^{n}$ like $K_{i, j}$ entails the result. The independence of these results from $\beta>0$ is obvious.

${ }^{5}$ If we had $g^{T} D \mathcal{F}(\mathbf{u})=0$ then $g^{T}=(D \mathcal{F}(\mathbf{u}))^{-1} 0=0$ which would contradict the fact that $g \in \mathcal{G}$.

${ }^{6}$ We use the following extension of the constant rank theorem, restated in our context (for details one can check [4, p. 96]). Let $f$ be a $\mathcal{C}^{s}$ application from an open set $\mathcal{O} \subset \mathbb{R}^{n}$ to $\mathbb{R}$. Assume that $D f(\mathbf{u})$ has constant rank $r$ for all $\mathbf{u} \in \mathcal{O}$. Given a $c \in \mathbb{R}$, the inverse image $f^{-1}(c)$ (supposed nonempty) is a $\mathcal{C}^{s}$-manifold of $\mathbb{R}^{n}$ of dimension $n-r$. 
Lamma 3. Let $(A, B) \in \mathbb{R}^{n \times n} \times \mathbb{R}^{n \times n}$ satisfy

$$
\begin{aligned}
& A=\operatorname{diag}\left(\left\{a_{i}\right\}_{i=1}^{n}\right) \text { where } a_{i}>0, \quad \forall i \in \mathbb{I}_{n}, \\
& B=B^{T}, \quad B \succeq 0 .
\end{aligned}
$$

Consider the $n \times n$ matrix $M \stackrel{\text { def }}{=}(A+B)^{-1} A$. Then all eigenvalues of $M^{T} M$ belong to $(0,1]$.

Proof: Let $\lambda$ be an eigenvalue of $(A+B)^{-1} A$ and $\mathbf{v} \in \mathbb{R}^{n} \backslash\{0\}$ a right eigenvector corresponding to $\lambda$. Then

$$
\begin{array}{rlrl}
\lambda \mathbf{v} & =M \mathbf{v} \\
\Leftrightarrow & & \lambda \mathbf{v} & =(A+B)^{-1} A \mathbf{v} \\
\Leftrightarrow & \lambda(A+B) \mathbf{v} & =A \mathbf{v} \\
\Leftrightarrow & \lambda B \mathbf{v} & =(1-\lambda) A \mathbf{v} .
\end{array}
$$

If $\lambda=0$ then (31) yields $A \mathbf{v}=0$ which is impossible because $A \succ 0, A^{T}=A$ and $\mathbf{v} \neq 0$. Hence

$$
\lambda \neq 0 .
$$

Furthermore, (31) yields

$$
\lambda \mathbf{v}^{T} B \mathbf{v}=(1-\lambda) \mathbf{v}^{T} A \mathbf{v} .
$$

Using that $A \succ 0$ and $B \succeq 0$, the last equation shows that

$$
\frac{1}{\lambda}-1=\frac{\mathbf{v}^{T} B \mathbf{v}}{\mathbf{v}^{T} A \mathbf{v}} \geq 0
$$

Combining the latter inequality with (32) entails that

$$
0<\lambda \leq 1 \text {. }
$$

Hence all eigenvalues of $M$ live in $(0,1]$.

Using that $A$ is diagonal with positive diagonal entries, we can write down

$$
A+B=A^{\frac{1}{2}}\left(I+A^{-\frac{1}{2}} B A^{-\frac{1}{2}}\right) A^{\frac{1}{2}} .
$$

Then the definition of $M$ shows that ${ }^{7}$

$$
\begin{aligned}
M^{T} M & =A(A+B)^{-2} A \\
& =\left(I+A^{-\frac{1}{2}} B A^{-\frac{1}{2}}\right)^{-2} .
\end{aligned}
$$

Using (35), the expression in (30) is equivalent to

$$
\begin{aligned}
\lambda \mathbf{v} & =A^{-\frac{1}{2}}\left(I+A^{-\frac{1}{2}} B A^{-\frac{1}{2}}\right)^{-1} A^{-\frac{1}{2}} A \mathbf{v} \\
\Leftrightarrow \quad \lambda\left(A^{\frac{1}{2}} \mathbf{v}\right) & =\left(I+A^{-\frac{1}{2}} B A^{-\frac{1}{2}}\right)^{-1}\left(A^{\frac{1}{2}} \mathbf{v}\right) .
\end{aligned}
$$

Combining the last result with (36) yields

$$
\begin{aligned}
M^{T} M\left(A^{\frac{1}{2}} \mathbf{v}\right) & =\left(I+A^{-\frac{1}{2}} B A^{-\frac{1}{2}}\right)^{-1}\left(I+A^{-\frac{1}{2}} B A^{-\frac{1}{2}}\right)^{-1}\left(A^{\frac{1}{2}} \mathbf{v}\right) \\
& =\lambda\left(I+A^{-\frac{1}{2}} B A^{-\frac{1}{2}}\right)^{-1}\left(A^{\frac{1}{2}} \mathbf{v}\right) \\
& =\lambda^{2}\left(A^{\frac{1}{2}} \mathbf{v}\right) .
\end{aligned}
$$

${ }^{7}$ Remind that $A=A^{T}$. 
Consequently, $\lambda^{2}$ is an eigenvalue of $M^{T} M$ corresponding to an eigenvector given by $\left(A^{\frac{1}{2}} \mathbf{v}\right)$. Thus all eigenvalues of $M^{T} M$ belong to $(0,1]$ as well.

\section{E. Proof of Proposition 2}

Let us denote

$$
\begin{aligned}
& A(\mathbf{u})=\operatorname{diag}\left(\left\{\psi^{\prime \prime}\left(\mathcal{F}_{i}(\mathbf{u})-\mathbf{u}[i]\right)\right\}_{i=1}^{n}\right) \\
& B(\mathbf{u})=G^{T} \operatorname{diag}\left(\left\{\phi^{\prime \prime}\left(\mathbf{g}_{i}^{T} \mathcal{F}(\mathbf{u})\right)\right\}_{i=1}^{n}\right) G
\end{aligned}
$$

as well as

$$
M(\mathbf{u})=(A(\mathbf{u})+\beta B(\mathbf{u}))^{-1} A(\mathbf{u})
$$

Then $D \mathcal{F}(\mathbf{u})=M(\mathbf{u})$. We have

$$
\mathcal{F}(\mathbf{u}+\boldsymbol{\zeta})-\mathcal{F}(\mathbf{u})=\int_{0}^{1} D \mathcal{F}(\mathbf{u}+t \boldsymbol{\zeta}) \boldsymbol{\zeta} d t=\int_{0}^{1} M(\mathbf{u}+t \boldsymbol{\zeta}) \boldsymbol{\zeta} d t
$$

Using Lemma 3 and $\mathrm{H} 1$, for any $\mathbf{v} \in \mathbb{R}^{n}$, all eigenvalues of $\left.(M(\mathbf{v}))^{T} M(\mathbf{v})\right)$ are in $(0,1]$. By the definition of the matrix 2-norm [37] one obtains

$$
t \in[0,1] \text { and } \boldsymbol{\zeta} \in \mathbb{R}^{n} \Rightarrow\|M(\mathbf{u}+t \boldsymbol{\zeta})\|_{2} \leq 1
$$

Noticing that $M(\cdot)$ is a continuous mapping, the mean value theorem (see e.g. [4], [14]) shows that

$$
\|\mathcal{F}(\mathbf{u}+\boldsymbol{\zeta})-\mathcal{F}(\mathbf{u})\|_{2} \leq \max _{0 \leq t \leq 1}\|M(\mathbf{u}+t \boldsymbol{\zeta})\|_{2}\|\boldsymbol{\zeta}\|_{2} \leq\|\boldsymbol{\zeta}\|_{2}
$$

\section{REFERENCES}

[1] T. Arici, S. Dikbas, and Y. Altunbasak. A histogram modification framework and its application for image contrast enhancement. IEEE Transactions on Image Processing, 18(9):1921-1935, 2009.

[2] G. Aubert and P. Kornprobst. Mathematical Problems in Image Processing, 2006, Springer-Verlag, Berlin, 2nd edition.

[3] A.N. Avanaki. Exact global histogram specification optimized for structural similarity. Optical Review, 16(6):613-621, 2009.

[4] A. Avez. Calcul Différentiel. Masson, 1991.

[5] F. Baus, M. Nikolova and G. Steidl, Fully smoothed $\ell_{1}-\mathrm{TV}$ models: Bounds for the minimizers and parameter choice, tech. report, hal-00722743, v2. (http://hal.archives-ouvertes.fr/hal-00722743.)

[6] J.-F. Bonnans, J.-C. Gilbert, C. Lemaréchal and C. Sagastizábal. Numerical optimization (theoretical and practical aspects) Springer (Berlin; New York NY; Hong Kong) 2003.

[7] J. Bioucas-Dias, M. Figueiredo, and R. Nowak. Total variation-based image deconvolution: a majorization-minimization approach. In Proc. IEEE International Conference on Acoustics, Speech and Signal Processing ICASSP 2006, volume 2, pages II-II, 14-19 May 2006.

[8] M. Black and A. Rangarajan. On the unification of line processes, outlier rejection, and robust statistics with applications to early vision. International Journal of Computer Vision, 19 (1996), pp. 57-91.

[9] N. G. De Bruijn, N. G. Some classes of integer-valued functions. Indag. Math., 17(3), 1955.

[10] P.-J. Cahen and J.-L. Chabert. Integer-valued Polynomials. vol. 48 of Mathematical Surveys and Monographs, Amer. Math. Soc., 1997.

[11] R. Chan, M. Nikolova, and Y. Wen. A variational approach for exact histogram specification. Lecture Notes in Computer Science series, 6667:preprint, 2011.

[12] S. Chen and A. R. Ramli. Contrast enhancement using recursive mean-separate histogram equalization for scalable brightness preservation. IEEE Transactions on Consumer Electronics, 49(4):1301-1309, 2003.

[13] H.D. Cheng, XH Jiang, Y. Sun, and J. Wang. Color image segmentation: advances and prospects. Pattern recognition, 34(12):2259-2281, 2001.

[14] P. G. Ciarlet. Introduction to Numerical Linear Algebra and Optimization, Cambridge University Press, 1989.

[15] D. Coltuc and P. Bolon. An inverse problem: Histogram equalization. In Signal Processing IX, Theories and Applications, EUSIPCO'98, volume 2, pages 861-4, 1998. 
[16] D. Coltuc and P. Bolon. Robust watermarking by histogram specification. In Image Processing, 1999. ICIP 99. Proceedings. 1999 International Conference on, volume 2, pages 236-239. IEEE, 1999.

[17] D. Coltuc, P. Bolon, and J.-M. Chassery. Exact histogram specification. IEEE Transactions on Image Processing, 15(5):11431152, 2006.

[18] I.J. Cox, S. Roy, and S.L. Hingorani. Dynamic histogram warping of image pairs for constant image brightness. In International Conference on Image Processing, volume 2, pages 366-369, 1995.

[19] J. Delon. Midway image equalization. Journal of Mathematical Imaging and Vision, 21(2):119-134, 2004.

[20] A. L. Dontchev and T. Zollezi. Well-posed Optimization Problems. Springer-Verlag, New York, 1993.

[21] L. C. Evans and R. F. Gariepy. Measure Theory and Fine Properties of Functions. Studies in Advanced Mathematics, CRC Press, Roca Baton, FL, 1992.

[22] S. Ferradans, G.-S. Xia, G. Peyré, and J.-F. Aujol. Optimal transport mixing of gaussian texture models, tech. report, Preprint Hal-00662720, 2012

[23] A. Fiacco and G. McCormic. Nonlinear Programming. Classics in Applied Mathematics, SIAM, Philadelphia, 1990.

[24] M. A. T. Figueiredo, J. M. Bioucas-Dias and R. D. Nowak. Majorization-minimization algorithms for wavelet-based image restoration. IEEE Trans. Image Process., 16(12):2980-2991, DEC 2007.

[25] D. Geman and G. Reynolds. Constrained restoration and the recovery of discontinuities. IEEE Trans. Pattern Anal. Mach. Intell., 14(3):367-383, 1992.

[26] D. Geman and C. Yang. Nonlinear image recovery with half-quadratic regularization. IEEE Trans. Image Process., 4(7):932-946, JUL 1995.

[27] R.C. Gonzalez and R.E. Woods. Digital Image Processing. Beijlng: Publishing House of Electronics Industry, 2005.

[28] Y. Gousseau and J. M. Morel. Are natural images of bounded variation? SIAM Journal on Mathematical Analysis, 33(3):634-648, 2001.

[29] E. L. Hall. Almost uniform distributions for computer image enhancement, IEEE Transactions on Computers, C-23 (1974), pp. 207-208.

[30] M. Hazewinkel, ed. Diophantine Equations. Encyclopaedia of Mathematics, Springer, 2001.

[31] D. Hunter and K. Lange. A tutorial on MM algorithms. Am. Stat., 58(1):30-37, 2004.

[32] A. K. Jain. Fundamental of Digital Image Processing. Prentice-Hall, 1989.

[33] H. T. Jongen, P. Jonker, and F. Twilt. Nonlinear Optimization in Finite Dimensions. Kluwer Academic Press, 1 ed., 1986.

[34] Y. Kim. Contrast enhancement using brightness preserving bi-histogram equalization. IEEE Transactions on Consumer Electronics, 43(1):1-8, 1997.

[35] J. Lafontaine. Introduction aux Variétés Différentielles. EDP sciences, France, 2 ed., 2010.

[36] D. Krishnan, P. Lin, and X. Tai. An efficient operator splitting method for noise removal in images. Commun. Comput. Phys., $1: 847-858,2006$.

[37] C. D. Meyer. Matrix Analysis and Applied Linear Algebra. SIAM, 2000.

[38] M. Mignotte. An energy based model for the image edge histogram specification problem, IEEE Trans. Image Process., 21 (2012), pp. 379-386.

[39] D. Mumford and A. Desolneux. Pattern Theory: The Stochastic Analysis of Real-World Signals, A. K. Peters Ltd., 2010.

[40] Y. Nesterov. Smooth minimization of non-smooth functions. Math. Program., Ser. A, 103(1):127-152, 2005.

[41] J. Portilla and E. P. Simoncelli. A parametric texture model based on joint statistics of complex wavelet coefficients, International Journal of Computer Vision, 40 (2000), pp. 49-71.

[42] M. Lysaker and X. Tai. Noise removal using smoothed normals and surface fitting. IEEE Trans. Image Process., 13(10):13451357, Oct. 2004.

[43] S. Narasimhan and S. Nayar. Contrast restoration of weather degraded images. IEEE Transactions on Pattern Analysis and Machine Intelligence, 25:713-724, 2003.

[44] K. Ni, X. Bresson, T. Chan, and S. Esedoglu. Local histogram based segmentation using the Wasserstein distance. International Journal of Computer Vision, 84(1):97-111, 2009.

[45] M. Nikolova. Local strong homogeneity of a regularized estimator. SIAM J. on Applied Mathematics, 61 (2000), pp. 633-658.

[46] M. Nikolova. A variational approach to remove outliers and impulse noise. Journal of Mathematical Imaging and Visio, 20(1-2):99-120, 2004.

[47] M. Nikolova. Weakly constrained minimization. Application to the estimation of images and signals involving constant regions. Journal of Mathematical Imaging and Vision, 21(2):155-175, 2004.

[48] M. Nikolova and M. Ng. Analysis of half-quadratic minimization methods for signal and image recovery. SIAM J. Sci. Comput., 2005.

[49] G. Pólya. Über ganzwertige ganze Funktionen. Rend. Circ. Mat. Palermo, 40 (1915), pp. 1-16.

[50] W. K. Pratt. Digital Image Processing. 2nd ed., Wiley, New York, 1991.

[51] A. Rosenfeld and K. Kak. Digital picture processing, Academic Press, Inc., P. H. Upper Saddle River, NJ, 1982.

[52] A.M. Reza. Realization of the contrast limited adaptive histogram equalization (CLAHE) for real-time image enhancement. The Journal of VLSI Signal Processing, 38(1):35-44, 2004. 
[53] J.-P Rolland, V. Vo, B. Bloss, and CK Abbey. Fast algorithms for histogram matching: Application to texture synthesis. Journal of Electronic Imaging, 9(1):39-45, 2000.

[54] L. Rudin, S. Osher, and E. Fatemi. Nonlinear total variation based noise removal algorithms. Physica D, 60:259-268, 1992.

[55] J. C. Russ. The Image Processing Handbook. 2nd ed., CRC Press, 1995.

[56] G. Sapiro and V. Caselles. Histogram modification via partial differential equations. In Image Processing, 1995. Proceedings., International Conference on, volume 3, pages 632-635. IEEE, 2002.

[57] D. Sen and P. Sankar. Automatic exact histogram specification for contrast enhancement and visual system based quantitative evaluation, IEEE Trans. Image Process., 20 (2011), pp. 1211-1220.

[58] K. Sim, C. Tso and Y. Tan. Recursive sub-image histogram equalization applied to gray scale images. Pattern Recognition Letters, 28(2007), pp. 1209-1221.

[59] T. Tan, K. Sim and C. Tso. Image enhancement using background brightness preserving histogram equalisation. Electronics Letters, 48(2012), pp. 150-U34.

[60] C. Vogel and M. Oman. Iterative method for total variation denoising. SIAM J. Sci. Comput., 17:227-238, 1996.

[61] Y. Wan and D. Shi. Joint exact histogram specification and image enhancement through the wavelet transform. IEEE Transactions on Image Processing, 16(9):2245-2250, 2007.

[62] C. Wang and Z. Ye. Brightness preserving histogram equalization with maximum entropy: a variational perspective. IEEE Transactions on Consumer Electronics, 51(4):1326-1334, 2005. 